\title{
Weak formulation of elastodynamics in domains with growing cracks
}

\author{
Emanuele Tasso $^{1}$ (D)
}

Received: 19 March 2019 / Accepted: 15 November 2019 / Published online: 25 November 2019 (c) Fondazione Annali di Matematica Pura ed Applicata and Springer-Verlag GmbH Germany, part of Springer Nature 2019

\begin{abstract}
In this paper, we formulate and study the system of elastodynamics on domains with arbitrary growing cracks. This includes homogeneous Neumann conditions on the crack sets and mixed general Dirichlet-Neumann conditions on the boundary. The only assumptions on the crack sets are to be $(n-1)$-rectifiable with finite surface measure, and increasing in the sense of set inclusions. In particular, they might be dense; hence, the weak formulation must fall outside the usual context of Sobolev spaces and Korn's inequality. We prove existence of a solution for both the damped and undamped systems, while in the damped case we are also able to prove uniqueness and an energy balance.
\end{abstract}

Keywords Second-order linear hyperbolic system - Dynamic fracture mechanics · Cracking domains $\cdot$ Boundary conditions $\cdot$ Bounded deformation

Mathematics Subject Classification 35L53 $\cdot 35 \mathrm{~A} 01 \cdot 35 \mathrm{~A} 02 \cdot 35 \mathrm{Q} 74 \cdot 26 \mathrm{~A} 45 \cdot 74 \mathrm{R} 10$

\section{Introduction}

The theory of dynamic fracture mechanics contains basically three principles that can be resumed as follows

- elastodynamics off the cracks;

- energy-dissipation balance which includes also the surface energy dissipated by the crack;

- a principle dictating when a crack must grow.

For the first two conditions, we refer to [13], while the third one is discussed in [14] in some more details and a maximal dissipation condition is proposed.

In this paper, we focus on the first issue. Precisely, we fix a time interval $[0, T]$ and we consider $\Omega \subset \mathbb{R}^{n}$ a regular domain as reference configuration, a fixed family of growing-intime crack sets $\Gamma(t)$ contained in $\Omega$, and $u(t, x)$ the displacement which might be essentially discontinuous for $x \in \Gamma(t)$. Then, given initial conditions, and mixed Dirichlet-Neumann

$凶 \quad$ Emanuele Tasso

emanueletss87@gmail.com; emanuele.tasso@tu-dresden.de

1 TU Dresden, Zellescher Weg 12-14, 01069 Dresden, Germany 
boundary conditions on $\partial \Omega$, we want to find a solution to the system of (possibly damped) elastodynamics

$$
\ddot{u}(t)-\operatorname{div}[\mathbb{C} \mathcal{E} u(t)]-\gamma \operatorname{div}[\mathbb{B} \mathcal{E} \dot{u}(t)]=f(t), \text { in } \Omega \backslash \Gamma(t)
$$

where $\mathbb{C}$ and $\mathbb{B}$ are the elasticity tensor and the viscosity tensor, respectively, $\mathcal{E} u$ denotes the symmetric part of the gradient of $u$, div denotes the divergence operator acting on the rows of matrices, $f(t)$ is a vector field representing the volume force, and at each time $t$ the system (1) is complemented with homogeneous Neumann condition on the crack $\Gamma(t)$. This last condition reflects the fact that no external forces are acting on the crack lips. The parameter $\gamma$ can take value only in $\{0,1\}$, and in particular, for $\gamma=1$ the system is called damped, while for $\gamma=0$ the system is called undamped.

In the corresponding quasi-static models, all the known existence results for the coupled problem $(u(t), \Gamma(t))$ without a priori assumptions on $\Gamma(t)$, are obtained by minimizing a weak form of the Griffith's energy on function spaces with no regularity on the jump sets except the $(n-1)$-rectifiability (see $[6,9,10,12])$. The existence of a solution with $\Gamma(t)$ closed is obtained only in particular cases through a regularity argument (see $[3,4])$. Therefore, also in the dynamic case we expect that in dealing with any general existence results, no a priori regularity assumptions on the crack sets $\Gamma(t)$ should be assumed. For this reason, we assume only that the cracks $\Gamma(t)$ are $(n-1)$-rectifiable with finite $(n-1)$-dimensional Hausdorff measure.

In this paper, we prove that in both the undamped and damped cases, a solution actually exists.

The first issue is to give a weak formulation to the system written in (1). The presence of the cracks forces at each time to solve the system on the set $\Omega \backslash \Gamma(t)$. Therefore, we need to introduce suitable function spaces $V_{t}$, containing for each time $t$ the solution $u(t)$ as well as the test functions. The scalar case, i.e., when (1) reduces to the wave equation, has been treated by Dal Maso, Larsen in [7]. Since the structure of the equation implies no bound on the amplitude of the jump of $u$, but only on the $L^{2}$-norm of the gradient

$$
\int_{\Omega \backslash \Gamma(t)}|\nabla u(t)|^{2} \mathrm{~d} x,
$$

they defined the problem in the context of $G S B V(\Omega)$ (for a definition, we refer to [1, Definition 4.26]). Precisely in [7], it has been shown the existence of a weak solution $u(t)$ living at each time $t$ in the space $G S B V_{2}^{2}(\Omega ; \Gamma(t))$, composed of all functions $u \in G S B V(\Omega) \cap L^{2}(\Omega)$ whose jump sets are contained in $\Gamma(t)$ and such that (2) is finite.

In our case, the structure of the equation leads to an estimate of

$$
\int_{\Omega \backslash \Gamma(t)}|\mathcal{E} u(t)|^{2} \mathrm{~d} x .
$$

Hence, $V_{t}$ needs to include all the displacements in $L^{2}\left(\Omega, \mathbb{R}^{n}\right)$ whose jump sets are contained in $\Gamma(t)$ and with square integrable symmetric gradient away form the cracks. Since we assume no regularity on the cracks, in this general context a Korn's type inequality is not true. This means that we cannot control the $L^{2}$-norm of the gradient of $u(t)$ with the $L^{2}$-norm of its symmetric part. As a consequence, we are forced to formulate our problem in the context of $B D$ functions and precisely to define $V_{t}=G S B D_{2}^{2}(\Omega ; \Gamma(t))$ (see Definition 2.3) and $V_{t}^{*}=$ $G S B D_{2}^{2}(\Omega ; \Gamma(t))^{*}$ its dual. Note that if $\Gamma(t)$ are closed sets in $\Omega$, then $G S B D_{2}^{2}(\Omega ; \Gamma(t))$ reduces to the space of square integrable vector fields, whose symmetric gradients in the sense of distribution on $\Omega \backslash \Gamma(t)$ are square integrable. 
The weak formulation of the system will be

$$
\langle\ddot{u}(t), \phi\rangle_{t}^{*}+\langle\mathbb{C} \mathcal{E} u(t), \mathcal{E} \phi\rangle_{H_{n}}+\langle\mathbb{B} \mathcal{E} \dot{u}(t), \mathcal{E} \phi\rangle_{H_{n}}=\langle f(t), \phi\rangle_{H} \forall \phi \in V_{t}
$$

for a.e. $t \in[0, T]$, where $\langle\cdot, \cdot\rangle_{t}^{*}$ denotes the duality pairing between $V_{t}$ and $V_{t}^{*},\langle\cdot, \cdot\rangle_{H}\langle\cdot, \cdot\rangle_{H_{n}}$ denote the scalar product in $L^{2}\left(\Omega, \mathbb{R}^{n}\right)$ and in $L^{2}\left(\Omega, \mathbb{M}_{\text {sym }}^{n \times n}\right)$, respectively.

We want to emphasize that one of the most serious mathematical issues arises because these spaces are varying (increasingly) in time, so that test functions at some time $t$ are not necessarily admissible test functions for times $s<t$. Moreover, since $u(t)$ lives on each time $t$ in different spaces $V_{t}$, we need to give a meaning to the second derivative in time $\ddot{u}(t)$ as an element of $V_{t}^{*}$.

While in [7] only homogeneous Neumann boundary condition was considered, in the present paper we consider also non-homogeneous mixed Dirichlet-Neumann boundary conditions on $\partial \Omega$. This introduces another difficulty when the crack sets approach the boundary, and as a consequence, possible problems may occur with the boundary conditions. Indeed, when we have non-homogeneous Neumann boundary condition on a part of $\partial \Omega$, we might think that when the elastic material between this part of the boundary and the crack sets is infinitesimally small, then the elastic reaction to the traction forces will be infinitesimal too. From a mathematical point of view, the difficulty is due to the lack of continuity of the trace operator acting on functions having jump sets close to the boundary. In order to solve this problem, we make use of the results obtained in [15], which allow us to restrict our attention to a suitable space of traction forces $F$.

We also show an energy balance and uniqueness for the damped problem. The energy balance we are able to prove in the damped case is a conservation of kinetic plus elastic plus dissipated energy due to the damping. For the undamped problem the energy balance, where only the kinetic plus the elastic energy are considered, is clearly false. This can be seen using the results of [2]. In the undamped case, the uniqueness is still an open problem.

This paper is organized as follows: in Sect. 2 we define the function spaces. Precisely, we define $V_{t}$, we show some functional properties about these spaces, and then we introduce the space of admissible traction forces $F$ appearing in the Neumann part of the boundary. Then we give a precise definition of $\ddot{u}(t)$ for a.e. $t \in[0, T]$ as an element of $V_{t}^{*}$, and we show that under some regularity assumptions on the test functions, $\ddot{u}$ satisfies an integration by parts formula in time.

In Sect. 3 we first give the definition of weak solution. Then we show an existence result for the damped equation complemented with boundary conditions, by a discrete in time approximation technique and passing to the limit when the time step goes to zero (see Theorem 3.2). More precisely to define the discrete approximate solution $u_{k}$ in the time interval $\left(t_{k}^{i}, t_{k}^{i+1}\right]$, suppose that we have already defined $u_{k}$ for $t \leq t_{k}^{i}$, and let $u_{k}^{i+1}$ be the minimizer in $V_{t_{i+1}}+w\left(t_{k}^{i}\right)^{1}$ of

$u \mapsto\left\|\frac{u-u_{k}^{i}}{\tau_{k}}-\frac{u_{k}^{i}-u_{k}^{i-1}}{\tau_{k}}\right\|^{2}+\langle\mathbb{C} \mathcal{E} u, \mathcal{E} u\rangle+\frac{1}{\tau_{k}}\left\langle\mathbb{B}\left(\mathcal{E} u-\mathcal{E} u_{k}^{i}\right), \mathcal{E} u-\mathcal{E} u_{k}^{i}\right\rangle-2\left\langle f_{k}^{i}, u\right\rangle$,

where $u_{k}^{i}=u_{k}\left(t_{k}^{i}\right),\|\cdot\|$ is the norm in $L^{2}$ and $\langle\cdot, \cdot\rangle$ is the $L^{2}$-scalar product, $f_{k}^{i}$ is a suitable discrete approximation of $f$ and $\tau_{k}$ is the time step. We define $u_{k}$ on $\left(t_{k}^{i}, t_{k}^{i+1}\right]$ as the linear interpolation between $u_{k}^{i}$ and $u_{k}^{i+1}$.

Then we show that the limit $u$ of the $u_{k}$ satisfies the energy balance (38). Precisely for each $k, u_{k}$ satisfies a discrete energy balance which converges to the desired energy balance

\footnotetext{
${ }^{1} V_{t_{i+1}}+w\left(t_{k}^{i}\right)$ is the space of functions that jump on $\Gamma\left(t_{i+1}\right)$ with Dirichlet boundary condition $w\left(t_{k}^{i}\right)$ on $\partial_{D} \Omega \subset \partial \Omega$.
} 
for $u$ as $k \rightarrow \infty$ (see Propositions 3.4 and 3.8). As a consequence, we deduce existence and uniqueness for the damped problem (see Theorem 3.7).

Finally, in Sect. 4 we show the existence of a weak solution to the undamped equation complemented with boundary conditions, always by a discrete in time approximation technique and passing to the limit when the time step goes to zero (Theorem 4.2).

\section{Notation and preliminary results}

We denote the space of $n \times n$ matrices with real entries as $\mathbb{M}^{n \times n}$ endowed with the Euclidean scalar product

$$
\xi \cdot \eta:=\sum_{j=1}^{n}\left(\sum_{i=1}^{n} \xi_{i j} \eta_{i j}\right),
$$

and we denote as $|\cdot|$ the associated norm; the subspace of symmetric $n \times n$ matrices is denoted by $\mathbb{M}_{s y m}^{n \times n} \cdot \mathcal{L}\left(\mathbb{M}^{n \times n}\right)$ is the space of continuous linear maps of $\mathbb{M}^{n \times n}$ into itself.

Let $\Omega \subset \mathbb{R}^{n}$ be an open set. We denote the space $L^{2}\left(\Omega, \mathbb{R}^{n}\right)$ as $H$, with scalar product $\langle\cdot, \cdot\rangle_{H}$ and with associated norm $\|\cdot\|_{H}$. Analogously we denote the space $L^{2}\left(\Omega, \mathbb{M}^{n \times n}\right)$ as $H_{n}$, with scalar product $\langle\cdot, \cdot\rangle_{H_{n}}$ and with associated norm $\|\cdot\|_{H_{n}}$.

Definition 2.1 We say that $\mathbb{C}: \Omega \rightarrow \mathcal{L}\left(\mathbb{M}^{n \times n}\right)$ is a bounded symmetric and positive definite tensor field, if it is $\mathcal{L}^{n}$-measurable and

- $\|\mathbb{C}\|_{L^{\infty}}<\infty$,

- $\mathbb{C}(x) \xi \in \mathbb{M}_{\text {sym }}^{n \times n}, \forall \xi \in \mathbb{M}^{n \times n}$, for a.e. $x \in \Omega$,

- $\mathbb{C}(x) \xi \cdot \eta=\xi \cdot \mathbb{C}(x) \eta, \forall \xi, \eta \in \mathbb{M}^{n \times n}$, for a.e. $x \in \Omega$ (symmetry),

- $\mathbb{C}(x) \xi \cdot \xi \geq \gamma_{0}|\xi|^{2}, \quad \forall \xi \in \mathbb{M}_{\text {sym }}^{n \times n}$, for a.e. $x \in \Omega\left(\gamma_{0}>0\right)$ (positiveness),

(which are the usual assumptions in linear elasticity). The strictly positive number $\gamma_{0}$ is called ellipticity constant of $\mathbb{C}$. Under the previous assumptions on $\mathbb{C}$, given any Lebesguemeasurable functions $\xi: \Omega \rightarrow \mathbb{M}^{n \times n}$, we write

$$
\|\xi\|_{H_{n}^{\mathbb{C}}}:=\int_{\Omega} \mathbb{C}(x) \xi(x) \cdot \xi(x) \mathrm{d} x .
$$

Remark 2.2 Thanks to the symmetry and positiveness properties of $\mathbb{C}$, it follows that the function $\|\cdot\|_{H_{n}^{\mathbb{C}}}$ defined on the real vector space of all measurable functions $\xi: \Omega \rightarrow \mathbb{M}_{\text {sym }}^{n \times n}$ is a norm. Moreover, by using also the $L^{\infty}$-bound, the norm $\|\cdot\|_{H_{n}^{\mathbb{C}}}$ is equivalent to the norm $\|\cdot\|_{H_{n}}$.

We recall that $G S B D_{2}^{2}(\Omega)$ is the space of vector fields $u \in G S B D(\Omega)$ (see [5] for the definition of $G S B D(\Omega)$ ) such that $u \in L^{2}\left(\Omega, \mathbb{R}^{n}\right)$, and their symmetric approximate gradients $\mathcal{E} u$ belong to $L^{2}\left(\Omega, \mathbb{M}_{\text {sym }}^{n \times n}\right)$.

Definition 2.3 Let $\Gamma \subset \Omega$ be a countably $\left(\mathcal{H}^{n-1}, n-1\right)$-rectifiable set (see [11, Definition 3.2.14]) with $\mathcal{H}^{n-1}(\Gamma)<\infty$. We define

$$
G S B D_{2}^{2}(\Omega ; \Gamma):=\left\{u \in G S B D_{2}^{2}(\Omega) \mid J_{u} \subset \Gamma\right\} .
$$


Proposition 2.4 Let $\Omega$ be an open set of $\mathbb{R}^{n}$, and let $\Gamma \subset \Omega$ be a countably $\left(\mathcal{H}^{n-1}, n-1\right)$ rectifiable set with $\mathcal{H}^{n-1}(\Gamma)<\infty$. Then the space $G S B D_{2}^{2}(\Omega ; \Gamma)$ endowed with the scalar product

$$
\langle u, v\rangle_{2}=\langle u, v\rangle_{H}+\langle\mathcal{E} u, \mathcal{E} v\rangle_{H_{n}},
$$

is a separable Hilbert space. Moreover, we denote by $\|\cdot\|$ the associated norm to the scalar product $\langle\cdot, \cdot\rangle_{2}$.

Proof Thanks to [5, Remark 4.6] we know that $G S B D(\Omega)$ is a real vector space, and as a consequence, $\operatorname{GSB}_{p}^{p}(\Omega)$ is a real vector space too. The fact that $G S B D_{p}^{p}(\Omega ; \Gamma)$ is also a real vector space follows once we prove that given $u, v \in G S B D(\Omega)$ then $J_{u+v} \subset J_{u} \cup J_{v}$ $\mathcal{H}^{n-1}$-a.e.. To see this, fix $\Xi$ an orthonormal basis of $\mathbb{R}^{n}$, say $\left\{\xi_{1}, \ldots, \xi_{n}\right\}$, and consider the directions in $\mathbb{S}^{n-1}$ defined by

$$
C(\Xi, \delta):=\left\{x \in \mathbb{S}^{n-1}|| x \cdot \xi_{i}|>(1 / \sqrt{n}-\delta)| x \mid, \text { for every } \xi_{i} \in \Xi\right\},
$$

where $\delta$ is any real number in $(0,1 / \sqrt{n})$. We claim that

$$
A:=\left\{x \in J_{u+v} \mid v_{u+v}(x) \in C(\Xi, \delta)\right\} \subset J_{u} \cup J_{v}, \mathcal{H}^{n-1} \text {-a.e. }
$$

Notice that for every $\xi \in \mathbb{S}^{n-1}$ and for every $y \in \xi^{\perp}$

$$
\left[\mathbb{R}^{n} \backslash\left(J_{u} \cup J_{v}\right)\right]_{y}^{\xi} \cap\left(J_{u}\right)_{y}^{\xi}=\emptyset \text {, and }\left[\mathbb{R}^{n} \backslash\left(J_{u} \cup J_{v}\right)\right]_{y}^{\xi} \cap\left(J_{v}\right)_{y}^{\xi}=\emptyset \text {, }
$$

and thanks to [5, Theorem 8.1] we deduce that for every $\xi \in \mathbb{S}^{n-1}$

$$
\left[\mathbb{R}^{n} \backslash\left(J_{u} \cup J_{v}\right)\right]_{y}^{\xi} \cap J_{\hat{u}_{y}^{\xi}}=\emptyset \text {, and }\left[\mathbb{R}^{n} \backslash\left(J_{u} \cup J_{v}\right)\right]_{y}^{\xi} \cap J_{\hat{v}_{y}^{\xi}}=\emptyset, \mathcal{H}^{n-1} \text {-a.e. } y \in \xi^{\perp} .
$$

Since the one-dimensional slices of $u$ and $v$ are $S B V_{l o c}$-functions, by (6) we deduce that for $\mathcal{H}^{n-1}$-a.e. $y \in \xi^{\perp}$ the sets $\left[\mathbb{R}^{n} \backslash\left(J_{u} \cup J_{v}\right)\right]_{y}^{\xi}$ is contained in the set of Lebesgue points of $\hat{u}_{y}^{\xi}+\hat{v}_{y}^{\xi}$ which in turn is contained in the set of Lebesgue points of $((u+v) \cdot \xi)_{y}^{\xi}$. By using again [5, Theorem 8.1], this means that for every $\xi \in \mathbb{S}^{n-1}$ we have

$$
\left[\mathbb{R}^{n} \backslash\left(J_{u} \cup J_{v}\right)\right]_{y}^{\xi} \cap\left(J_{u+v}^{\xi}\right)_{y}^{\xi}=\emptyset, \mathcal{H}^{n-1} \text {-a.e. } y \in \xi^{\perp} .
$$

Now suppose that (5) does not hold. This means that there exists a set $A^{\prime} \subset A$ with $\mathcal{H}^{n-1}\left(A^{\prime}\right)>0$ but $\mathcal{H}^{n-1}\left(\left(J_{u} \cup J_{v}\right) \cap A^{\prime}\right)=0$. Since $\Xi$ is a basis of $\mathbb{R}^{n}$, then there must exists $\xi_{i} \in \Xi$ such that

$$
\mathcal{H}^{n-1}\left(A^{\prime} \cap\left\{\left((u+v)^{+}-(u+v)^{-}\right) \cdot \xi_{i} \neq 0\right\}\right)>0 .
$$

By using also that for $\mathcal{H}^{n-1}$-a.e. $x \in A^{\prime} \cap\left\{\left((u+v)^{+}-(u+v)^{-}\right) \cdot \xi_{i} \neq 0\right\}$ we have $v_{u+v}(x) \cdot \xi_{i}>0$ (simply by definition of $A$ ), and by using Coarea formula applied to the projection map

$$
\pi^{\xi}: A^{\prime} \cap\left\{\left((u+v)^{+}-(u+v)^{-}\right) \cdot \xi_{i} \neq 0\right\} \rightarrow \xi^{\perp}
$$

we deduce that if we set $A_{i}^{\prime}:=A^{\prime} \cap\left\{\left((u+v)^{+}-(u+v)^{-}\right) \cdot \xi_{i} \neq 0\right\}$, then $\mathcal{H}^{n-1}\left(\pi^{\xi}\left(A_{i}^{\prime}\right)\right)>0$ and

$$
\mathcal{H}^{0}\left(\left[A^{\prime} \cap\left\{\left((u+v)^{+}-(u+v)^{-}\right) \cdot \xi_{i} \neq 0\right\}\right]_{y}^{\xi}\right)>0, \quad \mathcal{H}^{n-1} \text {-a.e. } y \in \pi^{\xi}\left(A_{i}^{\prime}\right) .
$$

But since $A_{i}^{\prime} \subset J_{u+v}^{\xi_{i}}$, by (7) this means also that

$$
\mathcal{H}^{0}\left(\left(J_{u} \cup J_{v}\right)_{y}^{\xi}\right)>0, \quad \mathcal{H}^{n-1} \text {-a.e. } y \in \pi^{\xi}\left(A_{i}^{\prime}\right),
$$


which is a contradiction since we assumed that $\mathcal{H}^{n-1}\left(\left(J_{u} \cup J_{v}\right) \cap A^{\prime}\right)=0$ and proves our claim. Finally, thanks to the arbitrariness of $\Xi$, relation (5) proves exactly that $J_{u+v} \subset J_{u} \cup J_{v}$ $\mathcal{H}^{n-1}$-a.e..

To prove the completeness, we argue in the following way. Suppose that $\left(u_{k}\right) \subset$ $G S B D_{p}^{p}(\Omega ; \Gamma)$ is a Cauchy sequence. In particular, $\left(u_{k}\right)_{k}$ converges strongly in $L^{p}\left(\Omega, \mathbb{R}^{n}\right)$ to some $u$. Since

$$
\sup _{k \in \mathbb{N}}\left(\left\|u_{k}\right\|_{L^{p}}+\left\|\mathcal{E} u_{k}\right\|_{L^{p}}+\mathcal{H}^{n-1}\left(J_{u_{k}}\right)\right)<\infty
$$

by Theorem [5, Theorem 11.3], eventually passing through a subsequence, we know that there exists $v \in G S B D(\Omega)$ such that $u_{k} \rightarrow v$ pointwise a.e. on $\Omega$ and $\mathcal{E} u_{k} \rightarrow \mathcal{E} v$ weakly in $L^{1}(\Omega)$. This implies $u=v$ and thanks to the lower semicontinuity of the $L^{p}$ norm with respect to the weak convergence also

$$
\|\mathcal{E} u\|_{L^{p}} \leq \liminf _{k \rightarrow \infty}\left\|\mathcal{E} u_{k}\right\|_{L^{p}}
$$

hence $u \in G S B D_{p}^{p}(\Omega)$.

It remains to prove that $J_{u} \subseteq \Gamma$. Using [5, Theorem, 11.3], for every open set $U \subset \Omega$ we have

$$
\mathcal{H}^{n-1}\left(J_{u} \cap U\right) \leq \liminf _{k \rightarrow \infty} \mathcal{H}^{n-1}\left(J_{u_{k}} \cap U\right) .
$$

Since the measure $\mathcal{H}^{n-1}\llcorner\Gamma$ is inner regular, for every $\epsilon>0$ we can find a compact set $K \subset \Gamma$, such that $\mathcal{H}^{n-1}(\Gamma \backslash K) \leq \epsilon$, and so

$$
\mathcal{H}^{n-1}\left(J_{u} \backslash K\right) \leq \liminf _{k \rightarrow \infty} \mathcal{H}^{n-1}\left(J_{u_{k}} \backslash K\right) \leq \liminf _{k \rightarrow \infty} \mathcal{H}^{n-1}(\Gamma \backslash K) \leq \epsilon .
$$

For the arbitrariness of $\epsilon$, we conclude that $\mathcal{H}^{n-1}\left(J_{u} \backslash \Gamma\right)=0$.

To prove the separability, consider the embedding $j: G S B D_{2}^{2}(\Omega ; \Gamma) \rightarrow L^{2}\left(\mathbb{R}^{n} \times \mathbb{R}^{n^{2}}\right)$ defined by $j(u):=(u, \mathcal{E} u)$. By the well-known fact that subspaces of a separable metric space are separable, since $j$ is an embedding, we deduce that also $G S B D_{2}^{2}(\Omega ; \Gamma)$ is separable.

The dual $G S B D_{2}^{2}(\Omega ; \Gamma)^{*}$ will not be identified with the underlying Hilbert space, but instead will be endowed with a pairing consistent with the $L^{2}$ inner product, as is usually done for the duals of Sobolev spaces. Since

$$
G S B D_{2}^{2}(\Omega ; \Gamma) \subset L^{2}\left(\Omega, \mathbb{R}^{n}\right)
$$

is a dense embedding, we have

$$
L^{2}\left(\Omega, \mathbb{R}^{n}\right)=L^{2}\left(\Omega, \mathbb{R}^{n}\right)^{*} \subset G S B D_{2}^{2}(\Omega ; \Gamma)^{*},
$$

and $L^{2}\left(\Omega, \mathbb{R}^{n}\right)$ is densely embedded in $G S B D_{2}^{2}(\Omega ; \Gamma)^{*}$.

In the case $\Omega$ has also finite perimeter, the trace operator $\operatorname{Tr}(\cdot)$ can be extended to the space $G S B D(\Omega ; \Gamma)$, using the notion of approximate limit on the point of the reduced boundary $\mathcal{F} \Omega$ (see [15, Definition 3.9]). Moreover, the following theorem holds true.

Theorem 2.5 Let $\Omega \subset \mathbb{R}^{n}$ be an open set of finite perimeter, and let $\Gamma \subset \Omega$ be a countably $\left(\mathcal{H}^{n-1}, n-1\right)$-rectifiable set, with $\mathcal{H}^{n-1}(\Gamma)<\infty$. Then there exists a measurable functions $\Theta: \mathcal{F} \Omega \rightarrow \mathbb{R}^{+}$such that

(a) $\mathcal{H}^{n-1}(\{\Theta=0\})=0$ and $\Theta \in L^{\infty}\left(\mathcal{F} \Omega, \mathcal{H}^{n-1}\right)$ (in particular, $\left.\|\Theta\|_{\infty} \leq 1\right)$; 
(b) For every $u \in G S B D_{2}^{2}(\Omega ; \Gamma)$ we have

$$
\int_{\mathcal{F} \Omega}|\operatorname{Tr}(u)|^{2} \Theta \mathrm{d} \mathcal{H}^{n-1} \leq C(n, 2)\left(\|u\|_{H}+\|\mathcal{E} u\|_{H_{n}}\right)^{2},
$$

where $C(n, 2)$ is a constant depending only on $n$ and 2.

Proof Let $\Theta^{+}$be the weight function given by [15, Theorem 3.2], if we define

$$
\Theta(x):=\Theta^{+}(x) \text { for } \mathcal{H}^{n-1} \text {-a.e. } x \in \mathcal{F} \Omega,
$$

then (a) and (b) are direct consequences of [15, Theorem 3.2] with $p=2$.

Now let $\partial_{N} \Omega$ be a Borel subset of $\mathcal{F} \Omega$. In order to impose a Neumann boundary condition in equation (1), we are led to study the continuity property of the following linear form

$$
u \mapsto \int_{\partial_{N} \Omega} F \cdot \operatorname{Tr}(u) \mathrm{d} \mathcal{H}^{n-1} u \in G S B D_{2}^{2}(\Omega ; \Gamma),
$$

where $F: \partial_{N} \Omega \rightarrow \mathbb{R}^{n}$ is some measurable vector field. In view of inequality (8) we introduce the following space.

Definition 2.6 (Admissible Neumann term) Let $\Omega, \Gamma$, and $\Theta$ be as in the previous theorem, and let $\partial_{N} \Omega$ be a Borel subset of $\mathcal{F} \Omega$. We define $N_{\Theta}:=L^{2}\left(\partial_{N} \Omega, \Theta \mathcal{H}^{n-1}\right)$, and we denote by $N_{\Theta}^{*}$ its dual. We identify $N_{\Theta}^{*}$ with the space of measurable vector fields $F: \partial_{N} \Omega \rightarrow \mathbb{R}^{n}$ such that

$$
\int_{\partial_{N} \Omega} \frac{|F|^{2}}{\Theta} \mathrm{d} \mathcal{H}^{n-1}<\infty
$$

and we consider the corresponding duality pairing between $N_{\Theta}^{*}$ and $N_{\Theta}$ given by

$$
\langle F, g\rangle_{\Theta}:=\int_{\partial_{N} \Omega} F \cdot g \mathrm{~d} \mathcal{H}^{n-1}\left(g \in N_{\Theta}\right) .
$$

The induced norm is denoted by $\|\cdot\|_{N_{\Theta}}^{*}$.

Putting together the definition of $N_{\Theta}$ and Theorem 2.5, we have the following result.

Proposition 2.7 Let $\Omega, \Gamma$, and $\Theta$ be as in Theorem 2.5. Let $\partial_{N} \Omega$ be a Borel subset of $\mathcal{F} \Omega$. If $F \in N_{\Theta}^{*}$ then the linear form defined in (9) belongs to $\operatorname{GSB} D_{2}^{2}(\Omega ; \Gamma)^{*}$.

Proof It is enough to use inequality (8) to have

$$
\int_{\partial_{N} \Omega} F \cdot \operatorname{Tr}(u) \mathrm{d} \mathcal{H}^{n-1} \leq\|F\|_{N_{\Theta}}^{*}\|\operatorname{Tr}(u)\|_{N_{\Theta}} \leq C(n, 2)\|F\|_{N_{\Theta}}^{*}\left(\|u\|_{H}+\|\mathcal{E} u\|_{H_{n}}\right) .
$$

Our choice of Neumann forces, in some sense, is natural. In fact looking at the construction of $\Theta$ made in [15], roughly speaking, it turns out that $\Theta$ measures the "closeness" of $\Gamma$ to the boundary. From a physical point of view, this might be interpreted as the fact that, when the elastic material between the Neumann boundary and the crack is infinitesimally small, then its elastic reaction can only balance traction forces which decrease their intensity (proportionally to $\Theta)$.

The following proposition is useful to prescribe a Dirichlet boundary condition on a certain portion of the boundary $\partial_{D} \Omega \subseteq \mathcal{F} \Omega$. 
Proposition 2.8 Let $\Omega, \Gamma$ be as in Proposition 2.7, let $\partial_{D} \Omega$ be a Borel subset of $\mathcal{F} \Omega$, and let $g: \partial_{D} \Omega \rightarrow \mathbb{R}$ be a measurable function. Then the set $\left\{u \in G S B D_{2}^{2}(\Omega ; \Gamma) \mid \operatorname{Tr}(u)=\right.$ $g$ on $\left.\partial_{D} \Omega\right\}$ is an affine closed subspace of $G S B D_{2}^{2}(\Omega ; \Gamma)$.

Proof The only non-trivial fact is to show that it is closed with respect to the norm induced by the scalar product (4). But this is a direct consequence of [15, Theorem 4.1].

Definition 2.9 Let $\partial_{D} \Omega \subseteq \mathcal{F} \Omega$ be a Borel set. We define

$$
G S B D_{2, D}^{2}(\Omega ; \Gamma):=\left\{u \in G S B D_{2}^{2}(\Omega ; \Gamma) \mid \operatorname{Tr}(u)=0 \text {, on } \partial \Omega_{D}\right\} .
$$

Thanks to our previous proposition, $G S B D_{2, D}^{2}(\Omega ; \Gamma)$ is actually an Hilbert space with scalar product inherited as a subspace of $G S B D_{2}^{2}(\Omega ; \Gamma)$.

Now fix $T>0$, and fix $\Gamma \subset \Omega$ a countably $\left(\mathcal{H}^{n-1}, n-1\right)$-rectifiable set with $\mathcal{H}^{n-1}(\Gamma)<$ $\infty$. Consider for $t \in[0, T]$ an increasing family of cracks $t \mapsto \Gamma(t)$

$$
\Gamma(s) \subseteq \Gamma(t) \subseteq \Gamma \text { if } s \leq t .
$$

For simplicity of notation, we denote $G S B D_{2}^{2}(\Omega ; \Gamma)$ by $V$ and $G S B D_{2, D}^{2}(\Omega ; \Gamma(t))$ by $V_{t}$. The norm in $V$ is denoted by $\|\cdot\|$, and the norm in $V_{t}$ with $\|\cdot\|_{t}$. Note that for $s<t$ we have $V_{s} \subset V_{t} \subset V$, and as we have already mentioned, since $V \subset H\left(\right.$ remember $H=L^{2}\left(\Omega, \mathbb{R}^{n}\right)$ ) is densely embedded in $H$, we have the embedding $H \subset V^{*}$ and the density of $H$ in $V^{*}$. Similarly $H$ is a dense subspace of $V_{t}^{*}$ for every $t \in[0, T]$. We denote the pairing between $V^{*}$ and $V$ by $\langle\cdot, \cdot\rangle$, and the associated dual norm $\|\cdot\|^{*}$, we denote the pairing between $V_{t}^{*}$ and $V_{t}$ by $\langle\cdot, \cdot\rangle_{t}$, and the associated dual norm $\|\cdot\|_{t}^{*}$. We note that these pairings are the unique continuous bilinear maps on $V^{*} \times V$ and $V_{t}^{*} \times V_{t}$ such that $\langle f, v\rangle=\langle f, v\rangle_{H}$ and $\left\langle f, v_{t}\right\rangle_{t}=\left\langle f, v_{t}\right\rangle_{H}$ whenever $f \in H, v \in V, v_{t} \in V_{t}$.

If $s<t$ then $V_{s}$ is not dense in $V_{t}$ and so $V_{t}^{*}$ is not embedded in $V_{s}^{*}$. Anyway we can introduce the projection operators from $V_{t}^{*}$ to $V_{s}^{*}$ in the following way.

Definition 2.10 Let $s<t$ and let $i: V_{s} \rightarrow V_{t}$ denote the embedding $V_{s} \subset V_{t}$. Let $f$ be an element of $V_{t}^{*}$. Then we define the projection map $P_{s t}$ of $V_{t}^{*}$ onto $V_{s}^{*}$ as

$$
\left\langle P_{s t} f, v_{s}\right\rangle_{s}:=\left\langle f, i\left(v_{s}\right)\right\rangle_{t} \text { for any } v_{s} \in V_{s} .
$$

Note that the projection maps defined above are continuous and in particular $\left\|P_{s t} f\right\|_{s}^{*} \leq$ $\|f\|_{t}^{*}$. When there is no misunderstanding, we omit the notation $P_{s t} f$, since the action of $f \in V_{t}^{*}$ on elements of $V_{s} \subset V_{t}$ is clear from the context.

Lemma 2.11 Let $u \in W^{1, \infty}(0, T ; H)$. Assume that there exists a positive function $g \in$ $L^{2}(0, T)$, such that for every $s, t \in[0, T]$ with $s<t$, we have

$$
u \in W^{2,2}\left(t, T ; V_{s}^{*}\right) \text { and }\|\ddot{u}(r)\|_{s}^{*} \leq g(r) \text { for a.e. } r \in(t, T) .
$$

Then there exists a set $E \subset[0, T]$ of full measure, such that for every $t \in E$ there exists $w(t) \in V_{t}^{*}$ with the following properties

$$
\|w(t)\|_{t}^{*} \leq \tilde{g}(t)
$$

where $\tilde{g}(t)=\lim \sup _{h \rightarrow 0^{+}} \frac{1}{h} \int_{t}^{t+h} g(r) d r$, and

$$
\lim _{h \rightarrow 0^{+}} \frac{\dot{u}(t+h)-\dot{u}(t)}{h}=w(t) \text {, weakly in } V_{t}^{*},
$$


and

$$
\lim _{h \rightarrow 0} \frac{\dot{u}(t+h)-\dot{u}(t)}{h}=w(t), \text { strongly in } V_{s}^{*} \text { for every } s<t .
$$

In particular, for every $s \in[0, T]$ the functions $t \mapsto u(t)$ and $t \mapsto P_{s t} w(t)$, considered as a functions from $(s, T)$ to $V_{s}^{*}$, belong, respectively, to $W^{2,2}\left(s, T ; V_{s}^{*}\right)$ and $L^{2}\left(s, T ; V_{s}^{*}\right)$, and satisfy $\ddot{u}(t)=P_{s t} w(t)$ in $V_{s}^{*}$ for a.e. $t \in(s, T)$.

Remark 2.12 Under the previous hypothesis on $u$, for every $t \in[0, T] \dot{u}(t)$ is a well-defined element of $H$. More precisely, the functions $\dot{u}:[0, T] \rightarrow H$ is weakly continuous, i.e., for every $t_{k} \rightarrow t \in[0, T]$ we have

$$
\dot{u}\left(t_{k}\right) \rightarrow \dot{u}(t) \text { weakly in } H, \text { as } k \rightarrow \infty .
$$

Indeed, thanks to our hypothesis $\dot{u}(t)$ is a well-defined element of $V_{0}^{*}$ for every $t \in[0, T]$ and $\dot{u}\left(t_{k}\right) \rightarrow \dot{u}(t)$ weakly in $V_{0}^{*}$ whenever $t_{k} \rightarrow t$ as $k \rightarrow \infty$. Moreover, since $\dot{u} \in L^{\infty}(0, T ; H)$, by using the lower semicontinuity of the norm $\|\cdot\|_{H}$ with respect to the weak convergence in $H$, and the fact that $H \subset V_{0}^{*}$ is an embedding, we deduce that actually $\dot{u}(t) \in H$ for every $t \in[0, T]$. This shows that $\dot{u}(t)$ is a well-defined element of $H$ for every $t \in[0, T]$. Finally, by arguing as before it is easy to see that $\dot{u}\left(t_{k}\right) \rightarrow \dot{u}(t)$ weakly in $H$ as $k \rightarrow \infty$.

Remark 2.13 In the proof of Lemma 2.11, we are able to show that the convergence in (14) holds when we consider the incremental quotients only for positive $h$.

In the proof of Lemma 2.11, we shall use the following result on increasing sequences of subspaces of separable Hilbert spaces proved in [5, Lemma 2.3].

Lemma 2.14 Let $\left\{X_{t} \mid t \in[0, T]\right\}$ be an increasing family of closed linear subspaces of a separable Hilbert space $X$. Then, there exists a countable set $S \subset[0, T]$ such that for all $t \in[0, T] \backslash S$, we have

$$
X_{t}=\overline{\bigcup_{s<t} X_{s}}
$$

Proof (Lemma 2.11) Let $D \subset[0, T]$ be a countably dense set. Choose $s \in D$, then thanks to (12) for a.e. $t>s$ there exists $\ddot{u}(t)$ as an element of $V_{s}^{*}$ and $\|\ddot{u}(t)\|_{s}^{*} \leq \tilde{g}(t)$. By the fact that $D$ is countable, we have a set $E^{\prime} \subset(0, T)$ of full measure, such that if $t \in E^{\prime} \ddot{u}(t)$ exists as an element of $V_{s}^{*}$ and $\|\ddot{u}(t)\|_{s}^{*} \leq \tilde{g}(t)$ for every $s \in(0, t) \cap D$. Moreover, by density, for any $s_{1}<t$ there exists $s_{2} \in D$ with $s_{1}<s_{2}<t$ and thanks to the continuity of the projection map $P_{s_{1} s_{2}}$, we have the relation between $\ddot{u}(t)$ computed in $V_{s_{2}}^{*}$ and in $V_{s_{1}}^{*}$, given by

$$
\lim _{h \rightarrow 0} \frac{\dot{u}(t+h)-\dot{u}(t)}{h}=\ddot{u}(t) \text { in } V_{s_{2}}^{*} \Rightarrow \lim _{h \rightarrow 0} \frac{\dot{u}(t+h)-\dot{u}(t)}{h}=P_{s_{1} s_{2}} \ddot{u}(t) \text { in } V_{s_{1}}^{*},
$$

This means that for every $t \in E^{\prime}$ and for every $s<t$

$$
\ddot{u}(t) \text { exists in } V_{s}^{*},\|\ddot{u}(t)\|_{s}^{*} \leq \tilde{g}(t),
$$

and precisely two derivatives computed on different $V_{s}^{*}$ are related by (17). Hence, for every $t \in E^{\prime}, \ddot{u}(t)$ is well defined as an element of $V_{s}^{*}$ for every $s<t$.

The previous bound in (18) implies in particular

$$
u \in W^{2,2}\left(s, T ; V_{s}^{*}\right) \text { for every } s \in(0, T) .
$$


Now define

$$
E:=E^{\prime} \cup\left\{t \in[0, T] \mid \overline{\bigcup_{s<t} V_{s}}=V_{t} \text { and } \tilde{g}(t)<\infty\right\} .
$$

By Lemma 2.14, Lebesgue's differentiation theorem, and the definition of $E^{\prime}$, it holds that $E$ has still full measure.

Now let $t \in E$. In order to prove (13), we notice that for every $\phi \in V_{t}$ there exists an increasing sequence $\left(s_{n}\right)_{n \in \mathbb{N}}$ converging to $t$, and a sequence of functions $\left(\phi_{s_{n}}\right)_{n \in \mathbb{N}}$ with $\phi_{s_{n}} \in V_{s_{n}}$ strongly converging to $\phi$ in $V_{t}$. Hence, we can define $w(t): V_{t} \rightarrow \mathbb{R}$ as

$$
\langle w(t), \phi\rangle_{t}:=\lim _{n \rightarrow \infty}\left\langle\ddot{u}(t), \phi_{s_{n}}\right\rangle_{s_{n}} \text { for every } \phi \in V_{t} .
$$

We have to show that the previous limit exists and does not depend on the approximating sequence $\left(\phi_{s_{n}}\right)_{n \in \mathbb{N}}$. It is enough to notice that if $n>m$ then

$$
\begin{aligned}
\left\langle\ddot{u}(t), \phi_{s_{n}}\right\rangle_{s_{n}}-\left\langle\ddot{u}(t), \phi_{s_{m}}\right\rangle_{s_{m}} & =\left\langle\ddot{u}(t), \phi_{s_{n}}-\phi_{s_{m}}\right\rangle_{s_{n}} \\
& \leq\|\ddot{u}(t)\|_{s_{n}}^{*}\left\|\phi_{s_{n}}-\phi_{s_{m}}\right\|_{s_{n}} \\
& \leq \tilde{g}(t)\left\|\phi_{s_{n}}-\phi_{s_{m}}\right\|_{t} .
\end{aligned}
$$

This defines a continuous linear functional on $V_{t}$ and moreover $\|w(t)\|_{t}^{*} \leq \tilde{g}(t)$. This is exactly (13).

To prove (14) let $t \in E$. We fix $\epsilon>0$ and $\phi \in V_{t}$, and then we can find $s<t$ and $\phi_{s} \in V_{s}$ such that $\left\|\phi_{s}-\phi\right\|_{t} \leq \epsilon$. Hence,

$$
\begin{aligned}
\lim _{h \rightarrow 0^{+}}\left\langle\frac{\dot{u}(t+h)-\dot{u}(t)}{h}-w(t), \phi\right\rangle_{t} & =\lim _{h \rightarrow 0^{+}}\left\langle\frac{\dot{u}(t+h)-\dot{u}(t)}{h}-w(t), \phi_{s}+\left(\phi-\phi_{s}\right)\right\rangle_{t} \\
& \leq \limsup _{h \rightarrow 0^{+}} \frac{1}{h} \int_{t}^{t+h}\left\langle\ddot{u}(r)-w(t), \phi-\phi_{s}\right\rangle_{t} d r \\
& \leq \limsup _{h \rightarrow 0+}\left(\frac{1}{h} \int_{t}^{t+h} g(r) d r+\tilde{g}(t)\right)\left\|\phi-\phi_{s}\right\|_{t} \\
& \leq 2 \tilde{g}(t) \epsilon,
\end{aligned}
$$

where we used the fact that $u \in W^{2,2}\left(t, T ; V_{t}^{*}\right)$ and the fundamental theorem of calculus. The arbitrariness of $\epsilon$ gives assertion (14) and concludes the proof.

Definition 2.15 Under the assumption of Lemma 2.11, the element $w(t)$ of $V_{t}^{*}$ defined in (14) for a.e. $t \in[0, T]$ is denoted by $\ddot{u}(t)$.

Lemma 2.16 Let $u$ be as in Lemma 2.11 and consider $\varphi \in L^{2}(0, T ; V) \cap W^{1,2}(0, T ; H)$ such that $\varphi(t) \in V_{t}$ for every $t \in[0, T]$. Then the map $t \mapsto\langle\dot{u}(t), \varphi(t)\rangle_{H}$ is absolutely continuous on $[0, T]$ and more precisely

$$
\left\langle\dot{u}\left(t_{2}\right), \varphi\left(t_{2}\right)\right\rangle_{H}-\left\langle\dot{u}\left(t_{1}\right), \varphi\left(t_{1}\right)\right\rangle_{H}=\int_{t_{1}}^{t_{2}}\langle\ddot{u}(\tau), \varphi(\tau)\rangle_{\tau}+\langle\dot{u}(\tau), \dot{\varphi}(\tau)\rangle_{\tau} \mathrm{d} \tau,
$$

for every $0 \leq t_{1}<t_{2} \leq T$.

Proof By Remark 2.12 we know that $t \mapsto \dot{u}(t)$ is weakly continuous in $H$. Therefore, since $t \mapsto \varphi(t)$ is strongly continuous in $H$, we deduce that $t \mapsto\langle\dot{u}(t), \varphi(t)\rangle_{H}$ is a continuous real valued map. 
First of all, we prove our assertion for $\varphi(\cdot)_{h}:=\varphi(\cdot-h)$ instead of $\varphi(\cdot)$. Fix any $t \in$ $[h, T-h]$. Since $\varphi(\cdot-h) \in V_{t}$ on the time interval $[t, t+h]$ and $u \in W^{2,2}\left(t, t+h ; V_{t}^{*}\right)$, we easily deduce that

$$
\left\langle\dot{u}\left(t_{2}\right), \varphi_{h}\left(t_{2}\right)\right\rangle_{H}-\left\langle\dot{u}\left(t_{1}\right), \varphi_{h}\left(t_{1}\right)\right\rangle_{H}=\int_{t_{1}}^{t_{2}}\left\langle\ddot{u}(\tau), \varphi_{h}(\tau)\right\rangle_{\tau}+\left\langle\dot{u}(\tau), \dot{\varphi}_{h}(\tau)\right\rangle_{\tau} \mathrm{d} \tau,
$$

for every $t_{1}, t_{2} \in(t, t+h)\left(t_{1}<t_{2}\right)$. Since $t$ was arbitrary and $\left\langle\dot{u}(\cdot), \varphi_{h}(\cdot)\right\rangle_{H}$ is continuous, we can actually obtain (25) for every $t_{1}, t_{2} \in[h, T-h]\left(t_{1}<t_{2}\right)$.

Finally thanks to the fact $\varphi \in W^{1,2}(0, T ; H)$ the left-hand side of $(25)$ converges to $\left\langle\dot{u}\left(t_{2}\right), \varphi\left(t_{2}\right)\right\rangle_{H}-\left\langle\dot{u}\left(t_{1}\right), \varphi\left(t_{1}\right)\right\rangle_{H}$ as $h \rightarrow 0^{+}$, while using also $\varphi \in L^{2}(0, T ; V)$ (in particular, the continuity of the translations in $L^{2}$ ), the right-hand side of (25) converges to $\int_{t_{1}}^{t_{2}}\langle\ddot{u}(\tau), \varphi(\tau)\rangle_{\tau}+\langle\dot{u}(\tau), \dot{\varphi}(\tau)\rangle_{\tau} \mathrm{d} \tau$ and we are done.

\section{The damped system of elastodynamics}

From now on we consider the following standing assumptions:

(a) $\Omega \subset \mathbb{R}^{n}$ is an open set of finite perimeter;

(b) $(\Gamma(t))_{t \in[0, T]}$ is an increasing family of crack sets:

$$
\Gamma(s) \subseteq \Gamma(t) \subseteq \Gamma \text { for } s<t,
$$

where $\Gamma \subseteq \Omega$ is a countably $\left(\mathcal{H}^{n-1}, n-1\right)$-rectifiable set with $\mathcal{H}^{n-1}(\Gamma)<\infty$;

(c) $\partial_{D} \Omega, \partial_{N} \Omega$ are two disjoint Borel subsets of $\mathcal{F} \Omega$, respectively the Dirichlet and the Neumann part of the reduced boundary, such that $\partial_{D} \Omega \cup \partial_{N} \Omega=\mathcal{F} \Omega$;

(d) $\mathbb{C}$ and $\mathbb{B}$ are bounded symmetric and positive definite tensor fields with ellipticity constant $\gamma_{0}$ and $\gamma_{1}$, respectively (see Definition 2.1).

In this section, we deal with the damped system of elastodynamics:

$$
\ddot{u}(t)-\operatorname{div}[\mathbb{C} \mathcal{E} u(t)]-\operatorname{div}[\mathbb{B} \mathcal{E} \dot{u}(t)]=f(t) .
$$

Now we shall give the precise definition of weak solution:

Definition 3.1 Assume (a), (b), (c) and (d). With the notation introduced in Sect. 2, let $f \in$ $L^{2}\left(0, T ; V^{*}\right)$, let $w \in W^{2,2}(0, T ; H) \cap W^{1,2}\left(0, T ; H^{1}(\Omega)^{n}\right)$ and let $F \in L^{2}\left(0, T ; N_{\Theta}\right)$ where $\Theta$ is the function relative to the crack set $\Gamma$ given by Theorem 2.5. We say that $u$ is a weak solution to (27) on the time dependent domain $t \mapsto \Omega \backslash \Gamma(t)$ with Dirichlet boundary condition $w(t)$ on $\partial_{D} \Omega$, Neumann boundary condition $F(t)$ on $\partial_{N} \Omega$ and homogeneous Neumann boundary condition on $\Gamma(t)$, if

$$
\begin{aligned}
& u \in W^{1, \infty}(0, T ; H) \cap W^{1,2}(0, T ; V) . \\
& \text { For every } t \in[0, T] u(t)-w(t) \in V_{t} . \\
& \text { For every } s \in[0, T) u \in W^{2,2}\left(s, T ; V_{s}^{*}\right) \text {, and } \\
& \left\|P_{s t} \ddot{u}(t)\right\|_{s}^{*} \leq g(t) \text { for a.e. } t \in(s, T) \text {, for some } g \in L^{2}(0, T) . \\
& \lim _{h \rightarrow 0^{+}} \int_{h}^{T} \frac{\|\dot{u}(t)-\dot{u}(t-h)\|_{H}^{2}}{h} \mathrm{~d} t=0 .
\end{aligned}
$$

For a.e. $t \in[0, T]$

$$
\langle\ddot{u}(t), \phi\rangle_{t}+\langle\mathbb{C} \mathcal{E} u(t), \mathcal{E} \phi\rangle_{H_{n}}+\langle\mathbb{B} \mathcal{E} \dot{u}(t), \mathcal{E} \phi\rangle_{H_{n}}-\langle F(t), \operatorname{Tr}(\phi)\rangle_{\Theta}
$$




$$
=\langle f(t), \phi\rangle_{t} \text {, for every } \phi \in V_{t}
$$

where $\ddot{u}(t)$ is the one given by Definition 2.15 .

Given $u^{0} \in V$ such that $u^{0}-w(0) \in V_{0}$ and $u^{1} \in H$, since $t \mapsto u(t)$ is strongly continuous in $V$ the initial value for $u$ is well defined as element of $V_{0}+w(0)$. Moreover, we are able to prescribe the initial conditions for $\dot{u}(0)$ asking

$$
\lim _{h \rightarrow 0^{+}} \frac{1}{h} \int_{0}^{h}\left\|\dot{u}(t)-u^{1}\right\|_{H}^{2} \mathrm{~d} t=0 .
$$

Since $\mathcal{E} u(t)$ and $\mathcal{E} \dot{u}(t)$ are in general only elements of $H_{n}$, it does not make sense to talk about their traces. For this reason the Neumann boundary conditions, $F(t)$ on $\partial_{N} \Omega$ and homogeneous on both sides of $\Gamma(t)$, have to be intended in a weak sense by means of integration by parts in Eq. (33). Moreover, condition (32) is technical and is related to the presence of the damping term. In fact, it plays a crucial role in view of the energy balance (38) (see Proposition 3.4).

The work of the external forces on the solution $u$ over a time interval $\left[t_{1}, t_{2}\right] \subset[0, T]$ is given by

$$
\mathcal{W}_{\text {load }}\left(u ; t_{1}, t_{2}\right):=\int_{t_{1}}^{t_{2}}\langle f(t), \dot{u}(t)\rangle_{t} \mathrm{~d} t
$$

which is well defined by (28) and the fact that $f \in L^{2}\left(0, T ; V^{*}\right)$. One would expect that the work on the solution $u$ due to the varying Dirichlet boundary conditions $w$ and Neumann boundary conditions $F$ over a time interval $\left[t_{1}, t_{2}\right] \subset[0, T]$ is given by

$$
\begin{aligned}
\mathcal{W}_{\text {bdry }}\left(u ; t_{1}, t_{2}\right):= & \int_{t_{1}}^{t_{2}}\left(\int_{\partial_{D} \Omega}(\mathbb{C} \mathcal{E} u(t)+\mathbb{B} \mathcal{E} u(t)) v \cdot \dot{w}(t) \mathrm{d} \mathcal{H}^{n-1}\right) \mathrm{d} t \\
& +\int_{t_{1}}^{t_{2}}\langle F(t), \dot{u}(t)\rangle_{\Theta} \mathrm{d} t
\end{aligned}
$$

Unfortunately, under the assumptions (28)-(31) the trace of the normal derivative $\partial_{\nu} u(t)$ cannot be defined, not even in a weaker sense, because $\mathcal{E} u(t)$ in general belongs only to $H_{n}$. We decide to solve this problem following [8, Proposition 3.1], by using the weak formulation of the work due to the Dirichlet boundary conditions:

$$
\begin{aligned}
& \mathcal{W}_{\text {bdry }}^{D}\left(u ; t_{1}, t_{2}\right):=\left\langle\dot{u}\left(t_{2}\right), \dot{w}\left(t_{2}\right)\right\rangle_{H}-\left\langle\dot{u}\left(t_{1}\right), \dot{w}\left(t_{1}\right)\right\rangle_{H}-\int_{t_{1}}^{t_{2}}\langle\ddot{w}(t), \dot{u}(t)\rangle_{H} \mathrm{~d} t \\
& \quad-\int_{t_{1}}^{t_{2}}\langle F(t), \dot{w}(t)\rangle_{\Theta} \mathrm{d} t-\int_{t_{1}}^{t_{2}}\langle f(t), \dot{w}(t)\rangle_{t} \mathrm{~d} t+\int_{t_{1}}^{t_{2}}\langle\mathbb{C} \mathcal{E} u(t)+\mathbb{B} \mathcal{E} \dot{u}(t), \mathcal{E} \dot{w}(t)\rangle_{H_{n}} \mathrm{~d} t .
\end{aligned}
$$

With this notation, the energy balance that we are able to prove for the solution $u$ to (27) has the following form:

$$
\begin{aligned}
& \frac{1}{2}\|\dot{u}(t)\|_{H}^{2}+\frac{1}{2}\|\mathcal{E} u(t)\|_{H_{n}^{\mathbb{C}}}^{2}+\frac{1}{2} \int_{0}^{t} \frac{1}{2}\|\mathcal{E} \dot{u}(\tau)\|_{H_{n}^{\mathbb{B}}}^{2} \mathrm{~d} \tau \\
& =\frac{1}{2}\left\|u^{1}\right\|_{H}^{2}+\frac{1}{2}\left\|\mathcal{E} u^{0}\right\|_{H_{n}^{\mathbb{C}}}+\mathcal{W}_{\text {load }}(u ; 0, t)+\mathcal{W}_{\text {bdry }}(u ; 0, t) .
\end{aligned}
$$

Now we prove the main result. 
Theorem 3.2 (Partial existence) Assume (a), (b), (c) and (d). Let $f, w$ and $F$ be as in Definition 3.1. Then, given two initial conditions $u^{0} \in V$ such that $u^{0}-w(0) \in V_{0}$ and $u^{1} \in H$, there exists a function $u$ satisfying (28)-(31) and (33) of Definition 4.1, with initial conditions $u(0)=u^{0}$ and (34). Moreover, $u$ satisfies the energy inequality

$$
\begin{aligned}
& \frac{1}{2}\|\dot{u}(t)\|_{H}^{2}+\frac{1}{2}\|\mathcal{E} u(t)\|_{H_{n}^{\mathbb{C}}}^{2}+\frac{1}{2} \int_{0}^{t} \frac{1}{2}\|\mathcal{E} \dot{u}(\tau)\|_{H_{n}^{\mathbb{B}}}^{2} \mathrm{~d} \tau \\
& \quad \leq \frac{1}{2}\left\|u^{1}\right\|_{H}^{2}+\frac{1}{2}\left\|\mathcal{E} u^{0}\right\|_{H_{n}^{\mathbb{C}}}+\mathcal{W}_{\text {load }}(u ; 0, t)+\mathcal{W}_{\text {bdry }}(u ; 0, t)
\end{aligned}
$$

for a.e. $t \in[0, T]$.

Remark 3.3 Since $F \in L^{2}\left(0, T ; N_{\Theta}\right)$, by Proposition 2.7 we have that $\langle F(t), \operatorname{Tr}(\phi)\rangle_{\Theta}$ is actually a duality pairing between $V_{t}^{*}$ and $V_{t}$. Therefore, we can absorb the Neumann term into the forcing term defining

$$
\langle\tilde{f}(t), \phi\rangle_{t}:=\langle f(t), \phi\rangle_{t}+\langle F(t), \operatorname{Tr}(\phi)\rangle_{\Theta},
$$

and we can reduce ourselves to prove Theorem 3.2 when (33) has the simplest form

$$
\langle\ddot{u}(t), \phi\rangle_{t}+\langle\mathbb{C} \mathcal{E} u(t), \mathcal{E} \phi\rangle_{H_{n}}+\langle\mathbb{B} \mathcal{E} \dot{u}(t), \mathcal{E} \phi\rangle_{H_{n}}=\langle f(t), \phi\rangle_{t} .
$$

Proof For $k \in \mathbb{N}$, we set $\tau_{k}:=T / k$ and $t_{k}^{j}:=j \tau_{k}$. For $j=1,2, \ldots, k$ we define $f_{k}^{j} \in V_{T}^{*}$ by

$$
f_{k}^{j}:=\frac{1}{\tau_{k}} \int_{t_{k}^{j}}^{t_{k}^{j+1}} f(\tau) \mathrm{d} \tau,
$$

and

$$
w_{k}^{j}:=w\left(t_{k}^{j}\right)
$$

(we use $w \in W^{1,2}\left(0, T ; H^{1}(\Omega)^{n}\right)$ implies that for every $t \in[0, T] w(t)$ is well defined in $\left.H^{1}(\Omega)^{n}\right)$.

Inductively, we define $u_{k}^{j}$ for $j=-1,0, \ldots, k$ by the following:

$$
u_{k}^{0}:=u^{0}, u_{k}^{-1}:=u^{0}-\tau_{k} u^{1}
$$

then, for $j=0,1, \ldots, k-1$, the function $u_{k}^{j+1}$ is the minimizer in $V_{t_{k}^{j+1}}+w_{k}^{j}$ of

$$
u \mapsto\left\|\frac{u-u_{k}^{j}}{\tau_{k}}-\frac{u_{k}^{j}-u_{k}^{j-1}}{\tau_{k}}\right\|_{H}^{2}+\|\mathcal{E} u\|_{H_{n}^{\mathbb{C}}}^{2}+\frac{1}{\tau_{k}}\left\|\left(\mathcal{E} u-\mathcal{E} u_{k}^{j}\right)\right\|_{H_{n}^{\mathbb{B}}}^{2}-2\left\langle f_{k}^{j}, u\right\rangle_{t_{k}^{j+1}} .
$$

Thanks to the ellipticity hypothesis on $\mathbb{C}$ and $\mathbb{B}$, at each step the above functional is coercive in $V_{t_{k}^{j+1}}+w_{k}^{j}$ because it is greater than

$$
c_{k}\left[\|u\|_{H}^{2}+\left(\gamma_{0}+\gamma_{1}\right)\|\mathcal{E} u\|_{H_{n}}^{2}\right]-2\left\|f_{k}^{j}\right\|_{t_{k}^{j+1}}^{*}\left(\|u\|_{H}+\|\mathcal{E} u\|_{H_{n}}\right)-a_{k}^{j+1}
$$

where $c_{k}:=\min \left\{1,1 / \tau_{k}^{2}\right\}, a_{k}^{j+1}$ is a constant depending only on $k j$, and $\gamma_{0}, \gamma_{1}>0$ are the ellipticity constants of $\mathbb{C}$ and $\mathbb{B}$, respectively. By using also that the first three terms in (43) are lower semicontinuous (here we use the symmetry and positiveness of $\mathbb{C}$ and $\mathbb{B}$ ) while the term $\left\langle f_{k}^{j}, u\right\rangle_{t_{k}^{j+1}}$ is even continuous with respect to the weak convergence in the closed 
affine subspace $V_{t_{k}^{j+1}}+w_{k}^{j+1}$, we deduce that the functional in (43) admits a minimizer $u_{k}^{j+1} \in V_{t_{k}^{j+1}}+w_{k}^{j+1}$. The Euler equation for the minimizer $u_{k}^{j+1}$ is

$$
\begin{aligned}
& \left\langle\frac{u_{k}^{j+1}-u_{k}^{j}}{\tau_{k}}-\frac{u_{k}^{j}-u_{k}^{j-1}}{\tau_{k}}, \frac{\phi}{\tau_{k}}\right\rangle_{H}+\left\langle\mathbb{C} \mathcal{E} u_{k}^{j+1}, \mathcal{E} \phi\right\rangle_{H_{n}}+\frac{1}{\tau_{k}}\left\langle\mathbb{B}\left(\mathcal{E} u_{k}^{j+1}-\mathcal{E} u_{k}^{j}\right), \mathcal{E} \phi\right\rangle_{H_{n}} \\
& \quad=\left\langle f_{k}^{j}, \phi\right\rangle_{t_{k}^{j+1}}
\end{aligned}
$$

for every $\phi \in V_{t_{k}^{j+1}}$. Then using $u_{k}^{j+1}-u_{k}^{j}-\left(w_{k}^{j+1}-w_{k}^{j}\right) \in V_{t_{k}^{j+1}}$ as $\phi$, we can write

$$
\begin{aligned}
& \left\|\frac{u_{k}^{j+1}-u_{k}^{j}}{\tau_{k}}\right\|_{H}^{2}-\left\langle\frac{u_{k}^{j+1}-u_{k}^{j}}{\tau_{k}}, \frac{u_{k}^{j}-u_{k}^{j-1}}{\tau_{k}}\right\rangle_{H} \\
& \quad-\left\langle\frac{u_{k}^{j+1}-u_{k}^{j}}{\tau_{k}}-\frac{u_{k}^{j}-u_{k}^{j-1}}{\tau_{k}}, \frac{w_{k}^{j+1}-w_{k}^{j}}{\tau_{k}}\right\rangle_{H} \\
& \quad+\left\|\mathcal{E} u_{k}^{j+1}\right\|_{H_{n}^{\mathbb{C}}}^{2}-\left\langle\mathbb{C} \mathcal{E} u_{k}^{j+1}, \mathcal{E} u_{k}^{j}\right\rangle_{H_{n}}-\left\langle\mathbb{C} \mathcal{E} u_{k}^{j+1}, \mathcal{E} w_{k}^{j+1}-\mathcal{E} w_{k}^{j}\right\rangle_{H_{n}} \\
& \quad+\frac{1}{\tau_{k}}\left\|\mathcal{E} u_{k}^{j+1}-\mathcal{E} u_{k}^{j}\right\|_{H_{n}}^{2} \\
& \quad-\frac{1}{\tau_{k}}\left\langle\mathbb{B}\left(\mathcal{E} u_{k}^{j+1}-\mathcal{E} u_{k}^{j}\right), \mathcal{E} w_{k}^{j+1}-\mathcal{E} w_{k}^{j}\right\rangle_{H_{n}}=\left\langle f_{k}^{j}, u_{k}^{j+1}-u_{k}^{j}\right\rangle_{t_{k}^{j+1}} \\
& -\left\langle f_{k}^{j}, w_{k}^{j+1}-w_{k}^{j}\right\rangle_{t_{k}^{j+1}} .
\end{aligned}
$$

Now using the identity $\|a\|^{2}-\langle a, b\rangle=\frac{1}{2}\|a\|^{2}+\frac{1}{2}\|a-b\|^{2}-\frac{1}{2}\|b\|^{2}$, multiplying by 2 , and rearranging, we get

$$
\begin{aligned}
& \left\|\frac{u_{k}^{j+1}-u_{k}^{j}}{\tau_{k}}\right\|_{H}^{2}+\left\|\frac{u_{k}^{j+1}-u_{k}^{j}}{\tau_{k}}-\frac{u_{k}^{j}-u_{k}^{j-1}}{\tau_{k}}\right\|_{H}^{2} \\
& \quad-2\left\langle\frac{u_{k}^{j+1}-u_{k}^{j}}{\tau_{k}}-\frac{u_{k}^{j}-u_{k}^{j-1}}{\tau_{k}}, \frac{w_{k}^{j+1}-w_{k}^{j}}{\tau_{k}}\right\rangle_{H} \\
& \quad+\left\|\mathcal{E} u_{k}^{j+1}\right\|_{H_{n}^{\mathbb{C}}}^{2}+\left\|\mathcal{E} u_{k}^{j+1}-\mathcal{E} u_{k}^{j}\right\|_{H_{n}^{\mathbb{C}}}^{2}+2\left\langle\mathbb{C} \mathcal{E} u_{k}^{j+1}, \mathcal{E} w_{k}^{j+1}-\mathcal{E} w_{k}^{j}\right\rangle_{H_{n}} \\
& \quad+2 \frac{1}{\tau_{k}}\left\|\mathcal{E} u_{k}^{j+1}-\mathcal{E} u_{k}^{j}\right\|_{H_{n}^{\mathbb{B}}}^{2}-2 \frac{1}{\tau_{k}}\left\langle\mathbb{B}\left(\mathcal{E} u_{k}^{j+1}-\mathcal{E} u_{k}^{j}\right), \mathcal{E} w_{k}^{j+1}-\mathcal{E} w_{k}^{j}\right\rangle_{H_{n}} \\
& =\left\|\frac{u_{k}^{j}-u_{k}^{j-1}}{\tau_{k}}\right\|_{H}^{2}+\left\|\mathcal{E} u_{k}^{j}\right\|_{H_{n}^{\mathbb{C}}}+2\left\langle f_{k}^{j}, u_{k}^{j+1}-u_{k}^{j}\right\rangle_{t_{k}^{j+1}}-2\left\langle f_{k}^{j}, w_{k}^{j+1}-w_{k}^{j}\right\rangle_{t_{k}^{j+1}} .
\end{aligned}
$$

Summing from $j=0$ to $i \in\{1, \ldots, k\}$ and using (42), we get

$$
\begin{aligned}
& \left\|\frac{u_{k}^{i+1}-u_{k}^{i}}{\tau_{k}}\right\|_{H}^{2}+\left\|\mathcal{E} u_{k}^{i+1}\right\|_{H_{n}^{\mathbb{C}}}^{2}+\sum_{j=0}^{i}\left\|\frac{u_{k}^{j+1}-u_{k}^{j}}{\tau_{k}}-\frac{u_{k}^{j}-u_{k}^{j-1}}{\tau_{k}}\right\|_{H}^{2} \\
& \quad+\sum_{j=0}^{i}\left\|\mathcal{E} u_{n}^{j+1}-\mathcal{E} u_{k}^{j}\right\|_{H_{n}^{\mathbb{C}}}^{2} \\
& \quad+2 \frac{1}{\tau_{k}} \sum_{j=0}^{i}\left\|\mathcal{E} u_{k}^{j+1}-\mathcal{E} u_{k}^{j}\right\|_{H_{n}^{\mathbb{B}}}^{2}=\left\|u^{1}\right\|_{H}^{2}+\left\|\mathcal{E} u^{0}\right\|_{H_{n}^{\mathbb{C}}}^{2}+2 \sum_{j=0}^{i}\left(\left\langle f_{k}^{j}, u_{k}^{j+1}-u_{k}^{j}\right\rangle_{t_{k}^{j+1}}\right.
\end{aligned}
$$




$$
\begin{aligned}
& +2 \sum_{j=0}^{i}\left\langle f_{k}^{j}, w_{k}^{j+1}-w_{k}^{j}\right\rangle_{t_{k}^{j+1}}+2 \sum_{j=0}^{i}\left\langle\mathbb{C} \mathcal{E} u_{k}^{j+1}+\frac{1}{\tau_{k}} \mathbb{B}\left(\mathcal{E} u_{k}^{j+1}-\mathcal{E} u_{k}^{j}\right), \mathcal{E} w_{k}^{j+1}-\mathcal{E} w_{k}^{j}\right\rangle_{H_{n}} \\
& -2 \sum_{j=0}^{i}\left\langle\frac{u_{k}^{j}-u_{k}^{j-1}}{\tau_{k}}, \frac{w_{k}^{j+1}-w_{k}^{j}}{\tau_{k}}-\frac{w_{k}^{j}-w_{k}^{j-1}}{\tau_{k}}\right\rangle_{H}+2\left\langle\frac{u_{k}^{i+1}-u_{k}^{i}}{\tau_{k}}, \frac{w_{k}^{i+1}-w_{k}^{i}}{\tau_{k}}\right\rangle_{H} \\
& -2\left\langle\frac{u_{k}^{1}-u_{k}^{0}}{\tau_{k}}, \frac{w_{k}^{1}-w_{k}^{0}}{\tau_{k}}\right\rangle_{H}
\end{aligned}
$$

We define the piecewise affine discrete approximations $u_{k}, v_{k}, w_{k}, z_{k}:[0, T] \rightarrow V$ for $t \in\left(t_{k}^{j}, t_{k}^{j+1}\right]$ by

$$
\begin{aligned}
u_{k}(t) & :=u_{k}^{j}+\frac{t-t_{k}^{j}}{\tau_{k}}\left(u_{k}^{j+1}-u_{k}^{j}\right), \\
w_{k}(t) & :=w_{k}^{j}+\frac{t-t_{k}^{j}}{\tau_{k}}\left(w_{k}^{j+1}-w_{k}^{j}\right), \\
v_{k}(t) & :=\frac{u_{k}^{j}-u_{k}^{j-1}}{\tau_{k}}+\frac{t-t_{k}^{j}}{\tau_{k}}\left(\frac{u_{k}^{j+1}-u_{k}^{j}}{\tau_{k}}-\frac{u_{k}^{j}-u_{k}^{j-1}}{\tau_{k}}\right), \\
z_{n}(t) & :=\frac{w_{k}^{j}-w_{k}^{j-1}}{\tau_{k}}+\frac{t-t_{k}^{j}}{\tau_{k}}\left(\frac{w_{k}^{j+1}-w_{k}^{j}}{\tau_{k}}-\frac{w_{k}^{j}-w_{k}^{j-1}}{\tau_{k}}\right),
\end{aligned}
$$

and the piecewise constant discrete approximations $\tilde{u}_{k}, \tilde{w}_{k}, f_{k}:[0, T] \rightarrow V$ for $t \in\left(t_{k}^{j}, t_{k}^{j+1}\right]$ by

$$
\tilde{u}_{k}(t):=u_{k}^{j+1}, \quad \tilde{w}_{k}(t):=w_{k}^{j+1}, \quad f_{k}(t):=f_{k}^{j} .
$$

Rewriting (48) in terms of $u_{k}, w_{k}, \tilde{u}_{k}, v_{k}, z_{k}$, we get the discrete energy balance for every $t \in\left(t_{k}^{j}, t_{k}^{j+1}\right)$ :

$$
\begin{aligned}
& \left\|\dot{u}_{k}(t)\right\|_{H}^{2}+\left\|\mathcal{E} u_{k}\left(t_{k}^{j+1}\right)\right\|_{H_{n}^{\mathbb{C}}}^{2}+\tau_{k} \int_{0}^{t_{k}^{j+1}}\left\|\dot{v}_{k}(\tau)\right\|_{H}^{2} \mathrm{~d} \tau+\tau_{k} \int_{0}^{t_{k}^{j+1}}\left\|\mathcal{E} \dot{u}_{k}(\tau)\right\|_{H_{n}^{\mathbb{C}}}^{2} \mathrm{~d} \tau \\
& \quad+\int_{0}^{t_{k}^{j+1}}\left\|\mathcal{E} \dot{u}_{k}(\tau)\right\|_{H_{n}^{\mathbb{B}}}^{2} \mathrm{~d} \tau=\left\|u^{1}\right\|_{H}^{2}+\left\|\mathcal{E} u^{0}\right\|_{H_{n}^{\mathbb{C}}}^{2}+2 \int_{0}^{t_{k}^{j+1}}\left\langle f_{k}(\tau), \dot{u}_{k}(\tau)\right\rangle_{t_{k}^{j+1}} \mathrm{~d} \tau \\
& \quad+2 \int_{0}^{t_{k}^{j+1}}\left\langle f_{k}(\tau), \dot{w}_{k}(\tau)\right\rangle_{t_{k}^{j+1}}+\left\langle\mathbb{C} \mathcal{E} \tilde{u}_{k}(\tau), \mathcal{E} \dot{w}_{k}(\tau)\right\rangle_{H_{n}}+\left\langle\mathbb{B} \mathcal{E} \dot{u}_{k}(\tau), \mathcal{E} \dot{w}_{k}(\tau)\right\rangle_{H_{n}} \mathrm{~d} \tau \\
& \quad-2 \int_{0}^{t_{k}^{j+1}}\left\langle\dot{u}_{k}(\tau), \dot{z}_{k}(\tau)\right\rangle_{H} \mathrm{~d} \tau+2\left\langle\dot{u}_{k}(t), \dot{w}_{k}(t)\right\rangle_{H}-2\left\langle u^{1}, \dot{w}_{k}(0)\right\rangle_{H}
\end{aligned}
$$

Let $M_{k}:=\sup _{t \in(0, T)}\left\|\dot{u}_{k}(t)\right\|_{H}, L_{k}:=\sup _{t \in(0, T)}\left\|\mathcal{E} \tilde{u}_{k}(t)\right\|_{H_{n}^{\mathbb{C}}}$. By (54) we can give the estimate

$$
M_{k}^{2}+L_{k}^{2}+\left\|\mathcal{E} \dot{u}_{k}\right\|_{L^{2}\left(0, T ; H_{n}^{\mathbb{B}}\right)}^{2} \leq a\left(M_{k}+L_{k}+\left\|\mathcal{E} \dot{u}_{k}\right\|_{L^{2}\left(0, T ; H_{n}^{\mathbb{B}}\right)}\right)+b,
$$

where $a$ and $b$ are constants that depend only on $\|f\|_{L^{2}\left(0, T ; V^{*}\right)},\|w\|_{W^{1,2}(0, T ; V)}$, $\|w\|_{W^{2,2}(0, T ; H)},\left\|u_{1}\right\|_{H}$ and on $T$. As a consequence, we can deduce the following

$\mathcal{E} u_{k}(t)$ and $\mathcal{E} \tilde{u}_{k}(t)$ are bounded in $H_{n}$ uniformly in $t$ and $k$,

$\dot{u}_{k}(t)$ and $v_{k}(t)$ are bounded in $H$ uniformly in $t$ and $k$, 
$\mathcal{E} \dot{u}_{k}$ is bounded in $L^{2}\left(0, T ; H_{n}\right)$ uniformly in $k$.

Notice also that $u^{0} \in H$ implies that $u_{k}$ is bounded in $H$ uniformly in $t$ and $k$. This together with (56) gives

$$
u_{k}(t) \text { is bounded in } V \text { uniformly in } t \text { and } k \text {. }
$$

Furthermore, using (49)-(51) and (53), we can rewrite (45) for all $t \in\left(t_{k}^{j}, t_{k}^{j+1}\right)$ as

$$
\left\langle\dot{v}_{k}(t), \phi\right\rangle_{H}+\left\langle\mathbb{C} \mathcal{E} \tilde{u}_{k}(t), \mathcal{E} \phi\right\rangle_{H_{n}}+\left\langle\mathbb{B} \mathcal{E} \dot{u}_{k}(t), \mathcal{E} \phi\right\rangle_{H_{n}}=\left\langle f_{k}(t), \phi\right\rangle_{t_{k}^{j+1}}
$$

for every $\phi \in V_{t_{k}^{j+1}}$. The last equation leads us to write for all $t \in\left(t_{k}^{j}, t_{k}^{j+1}\right)$

$$
\left\|\dot{v}_{k}(t)\right\|_{t_{k}^{j+1}}^{*} \leq\left\|\mathcal{E} \tilde{u}_{k}(t)\right\|_{H_{n}^{\mathbb{C}}}+\left\|\mathcal{E} \dot{u}_{k}(t)\right\|_{H_{n}^{\mathbb{B}}}+\left\|f_{k}(t)\right\|_{t_{k}^{j+1}}^{*} .
$$

In particular, fix $s \in[0, T)$, then for every $t_{1}, t_{2} \in[s, T]$ with $t_{1}<t_{2}$, we have

$$
\int_{t_{1}}^{t_{2}}\left\|P_{s t} \dot{v}_{k}(t)\right\|_{s}^{*} \mathrm{~d} t \leq \int_{t_{1}}^{t_{2}}\left(\left\|\mathcal{E} \tilde{u}_{k}(t)\right\|_{H_{n}^{\mathbb{C}}}+\left\|\mathcal{E} \dot{u}_{k}(t)\right\|_{H_{n}^{\mathbb{B}}}+\left\|f_{k}(t)\right\|_{s}^{*}\right) \mathrm{d} t .
$$

Using (56)-(58), and (61), there exists a constant $M$ such that eventually passing through a subsequence (depending on $s \in[0, T)$ ), if we call $v$ a weak limit of $v_{k}$ in $W^{1,2}\left(s, T ; V_{s}^{*}\right)$, and $\tilde{g}$ a weak limit of $t \mapsto\left\|\mathcal{E} \dot{u}_{k}(t)\right\|_{H_{n}}$ in $L^{2}(0, T)$, we have

$$
\int_{t_{1}}^{t_{2}}\left\|P_{s t} \dot{v}(t)\right\|_{s}^{*} \mathrm{~d} t \leq M\left|t_{2}-t_{1}\right|+\int_{t_{1}}^{t_{2}}\left(\tilde{g}(t)+\|f(t)\|_{s}^{*}\right) \mathrm{d} t,
$$

for every $t_{1}, t_{2} \in[s, T]$ with $t_{1}<t_{2}$.

Now if we fix a dense set $D \subset[0, T]$ by using a diagonal argument, we obtain a subsequence, not relabeled, such that

$$
\begin{aligned}
& u_{k} \rightarrow u \text { weakly in } W^{1,2}(0, T ; V), \\
& v_{k} \rightarrow v \text { weakly in } L^{2}(0, T ; H), \\
& v_{k} \rightarrow v \text { weakly in } W^{1,2}\left(s, T ; V_{s}^{*}\right) \text { for every } s \in D,
\end{aligned}
$$

and

$$
\int_{t_{1}}^{t_{2}}\left\|P_{s t} \dot{v}(t)\right\|_{s}^{*} \mathrm{~d} t \leq M\left|t_{2}-t_{1}\right|+\int_{t_{1}}^{t_{2}}\left(\tilde{g}(t)+\|f(t)\|_{s}^{*}\right) \mathrm{d} t,
$$

for every $t_{1}, t_{2} \in[s, T]$ with $t_{1}<t_{2}$.

Moreover, by using the continuity of the projection maps $P_{s t}$, it follows that (66) and (67) become

$$
v_{k} \rightarrow v \text { weakly in } W^{1,2}\left(s, T ; V_{s}^{*}\right) \text { for every } s \in[0, T),
$$

and

$$
\int_{t_{1}}^{t_{2}}\left\|P_{s t} \dot{v}(t)\right\|_{s}^{*} \mathrm{~d} t \leq M\left|t_{2}-t_{1}\right|+\int_{t_{1}}^{t_{2}}\left(\tilde{g}(t)+\|f(t)\|_{s}^{*}\right) \mathrm{d} t,
$$

for every $s \in[0, T)$ and every $t_{1}, t_{2} \in[s, T]$ with $t_{1}<t_{2}$. In particular,

$$
\left\|P_{s t} \dot{v}(t)\right\|_{s}^{*} \leq M+\tilde{g}(t)+\|f(t)\|_{s}^{*},
$$

for every $s \in[0, T)$ and a.e. $t>s$. 
By (57) it is easy to see that in fact

$$
u \in W^{1, \infty}(0, T ; H),
$$

and this with convergence (64) gives (28).

Now we want to show

$$
\dot{u}(t)=v(t) \text { in } H \text { for a.e. } t \in[0, T] .
$$

First of all, for $t \in\left(t_{k}^{j}, t_{k}^{j+1}\right)$ we have $\dot{u}_{k}(t)=v_{k}\left(t_{k}^{j+1}\right)$ and so

$$
\left\|\dot{u}_{k}(t)-v_{k}(t)\right\|_{t_{k}^{j+1}}^{*}=\left\|v_{k}\left(t_{k}^{j+1}\right)-v_{k}(t)\right\|_{t_{k}^{j+1}}^{*} \leq \int_{t_{k}^{j}}^{t_{k}^{j+1}}\left\|\dot{v}_{k}(\tau)\right\|_{t_{k}^{j+1}}^{*} \mathrm{~d} \tau \leq \tau_{k}^{\frac{1}{2}} C,
$$

where $C$ is a uniform bound on the $L^{2}$ norm of the right-hand side of (61). Then for all $s<t$ we have $\left\|\dot{u}_{k}(t)-v_{k}(t)\right\|_{s}^{*} \leq \tau_{k}^{\frac{1}{2}} C$, and this together with (65) implies $\dot{u}_{k} \rightarrow v$ weakly in $L^{2}\left(s, T ; V_{s}^{*}\right)$ for any $s \in[0, T)$. But also $\dot{u}_{k} \rightarrow \dot{u}$ weakly in $L^{2}(0, T ; H)$ by (64). So $v(t)=\dot{u}(t)$ in $V_{s}^{*}$, for every $s \in[0, T)$ and for a.e. $t \in(s, T)$. Since $v(t)$ and $\dot{u}(t)$ belong to $H$, and $H$ is embedded in $V_{s}^{*}$ for every $s \in[0, T]$, we finally get that $v(t)=\dot{u}(t)$ as elements of $H$ for a.e. $t \in[0, T]$. This together with (64) and (69) allows to conclude that

$$
u \in W^{2,2}\left(t, T ; V_{s}^{*}\right) \text { for every } s, t \in[0, T] \text { with } s<t .
$$

Let $g(t):=M+\tilde{g}(t)+\|f(t)\|_{t}^{*}$ then by (70) we have

$$
\left\|P_{s t} \ddot{u}(t)\right\|_{s}^{*} \leq g(t),
$$

for every $s \in[0, T)$ and for a.e. $t>s$, and we obtain (31).

Now we investigate the convergence of the constant piecewise interpolated $\tilde{u}_{k}$. Since by (64) $u_{k}$ are Lipschitz with values in $H$ uniformly in $k$, as before we get that

$$
\tilde{u}_{k} \rightarrow u \text { weakly in } L^{2}(0, T ; H),
$$

and since by (56) $\mathcal{E} \tilde{u}_{k}$ is bounded in $L^{2}\left(0, T ; H_{n}\right)$, we also obtain that up to subsequences

$$
\tilde{u}_{k} \rightarrow u \text { weakly in } L^{2}(0, T ; V) .
$$

Furthermore, note that $\tilde{u}_{k}\left(t-\tau_{k}\right)-\tilde{w}_{k}\left(t-\tau_{k}\right) \in V_{t}$ for every $t \in[0, T]$, and by (64) we can write for every $t \in\left(t_{k}^{j}, t_{k}^{j+1}\right]$

$$
\left\|u_{k}(t)-\tilde{u}_{k}\left(t-\tau_{k}\right)\right\|_{V}=\left\|u_{k}(t)-u_{k}\left(t_{k}^{j}\right)\right\|_{V} \leq \int_{t_{k}^{j}}^{t_{k}^{j+1}}\left\|\dot{u}_{k}(\tau)\right\|_{V} \mathrm{~d} \tau \leq \tau_{k}^{1 / 2} C,
$$

where $C$ is a constant independent on $k$. This means that, by using also $w \in W^{1,2}\left(0, T ; H^{1}\right.$ $\left.\left(\Omega ; \mathbb{R}^{n}\right)\right)$, we can write

$$
\tilde{u}_{k}\left(\cdot-\tau_{k}\right)-\tilde{w}_{k}\left(\cdot-\tau_{k}\right) \rightarrow u-w \text { weakly in } L^{2}(0, T ; V) .
$$

Since the linear subspace $\left\{v \in L^{2}(0, T ; V) \mid v(t) \in V_{t}\right.$ for a.e. $\left.t \in[0, T]\right\}$ is strongly closed in $L^{2}(0, T ; V)$, it is also weakly closed in $L^{2}(0, T ; V)$. Therefore, $u(t) \in V_{t}+w(t)$ for a.e. $t \in[0, T]$. Moreover, for every $t \in(0, T]$ there exists an increasing sequence $t_{i} \in[0, T]$ converging to $t$ such that $u\left(t_{i}\right)-w\left(t_{i}\right) \in V_{t_{i}}$ for every $i$. Thanks to (64) we know that $t \mapsto u(t)-w(t)$ is a strongly continuous map with values in $V$, and we obtain $u(t)-w(t) \in V_{t}$ for every $t \in(0, T]$. Together with the initial condition $u(0)=u^{0} \in V_{0}$ 
we obtain (29). Moreover, thanks to (74) and (75) we are in position to apply Lemma 2.14 to the function $u-w$ and hence to deduce that for a.e. $t \in[0, T]$

$$
\frac{\dot{u}(t+h)-\dot{u}(t)}{h} \rightarrow \ddot{u}(t) \text { weakly in } V_{t}^{*} \text { as } h \rightarrow 0^{+} .
$$

Now we want to show that (33) holds for a.e. $t \in[0, T]$ for every $\phi \in V_{t}$. We claim that there exists a negligible set $W \subset[0, T]$ such that for $s \in D$ and for all $\phi \in V_{s}$, we have

$$
\langle\ddot{u}(t), \phi\rangle_{s}+\langle\mathbb{C} \mathcal{E} u(t), \mathcal{E} \phi\rangle_{H_{n}}+\langle\mathbb{B} \mathcal{E} \dot{u}(t), \mathcal{E} \phi\rangle_{H_{n}}=\langle f(t), \phi\rangle_{s},
$$

for every $t \in(s, T] \backslash W$.

To prove the claim, first we fix $s \in D$ and $\phi \in V_{s}$. Using (60) we have for a.e. $t>s$

$$
\left\langle\dot{v}_{k}(t), \phi\right\rangle_{s}+\left\langle\mathbb{C} \mathcal{E} \tilde{u}_{k}(t), \mathcal{E} \phi\right\rangle_{H_{n}}+\left\langle\mathbb{B} \mathcal{E} \dot{u}_{k}(t), \mathcal{E} \phi\right\rangle_{H_{n}}=\left\langle f_{k}(t), \phi\right\rangle_{s} .
$$

Hence, we have also

$$
\int_{s}^{T}\left(\left\langle\dot{v}_{k}(t), \phi\right\rangle_{s}+\left\langle\mathbb{C} \mathcal{E} \tilde{u}_{k}(t), \mathcal{E} \phi\right\rangle_{H_{n}}+\left\langle\mathbb{B} \mathcal{E} \dot{u}_{k}(t), \mathcal{E} \phi\right\rangle_{H_{n}}-\left\langle f_{k}(t), \phi\right\rangle_{s}\right) \mathrm{d} t=0 .
$$

By construction $f_{k} \rightarrow f$ strongly in $L^{2}\left(0, T ; V^{*}\right)$. We know that $\dot{v}_{k} \rightarrow \dot{v}$ weakly in $L^{2}\left(s, T ; V_{s}^{*}\right)$ by $(125)$. Since $\dot{u}=v$ in $W^{1,2}\left(s, T ; V_{s}^{*}\right)$, we also have that $\ddot{u}=\dot{v}$ in $L^{2}\left(s, T ; V_{s}^{*}\right)$. Using also (64) and (77), we can pass to the limit in (82) to have

$$
\int_{s}^{T}\left(\langle\ddot{u}(t), \phi\rangle_{s}+\langle\mathbb{C} \mathcal{E} u(t), \mathcal{E} \phi\rangle_{H_{n}}+\langle\mathbb{B} \mathcal{E} \dot{u}(t), \mathcal{E} \phi\rangle_{H_{n}}-\langle f(t), \phi\rangle_{s}\right) \mathrm{d} t=0 .
$$

By $(81)$ we deduce that the integrand in (83) is zero for a.e. $t>s$. Since $V_{s}$ is separable, the set $N_{s}$ of $t>s$ for which (81) does not hold can be taken independent of $\phi$. We set $W$ to be the union over $s \in D$ of the sets $N_{s}$, so that $W$ also has measure zero. It follows that for every $s \in D$ and for every $t \in(s, T] \backslash W$ (80) holds, and this shows the claim.

Using Lemma 2.14, it follows that for a.e. $t$ and for every $\phi \in V_{t}$, there exist $s_{i} \nearrow t$ with $s_{i} \in D$ and $\phi_{i} \in V_{s_{i}}$, such that $\phi_{i} \rightarrow \phi$ strongly in $V_{t}$. Now note that if $t$ belongs also to $(0, T] \backslash W$, by our previous claim we have

$$
\begin{aligned}
& \left\langle\ddot{u}(t), \phi_{i}\right\rangle_{t}+\left\langle\mathbb{C} \mathcal{E} u(t), \mathcal{E} \phi_{i}\right\rangle_{H_{n}}+\left\langle\mathbb{B} \mathcal{E} \dot{u}(t), \mathcal{E} \phi_{i}\right\rangle_{H_{n}}-\left\langle f(t), \phi_{i}\right\rangle_{t} \\
& \quad=\left\langle\ddot{u}(t), \phi_{i}\right\rangle_{s_{i}}+\left\langle\mathbb{C} \mathcal{E} u(t), \mathcal{E} \phi_{i}\right\rangle_{H_{n}}+\left\langle\mathbb{B} \mathcal{E} \dot{u}, \mathcal{E} \phi_{i}\right\rangle_{H_{n}}-\left\langle f(t), \phi_{i}\right\rangle_{s_{i}}=0 .
\end{aligned}
$$

The convergence of the $\phi_{i}$ to $\phi$ gives (33).

Since by construction

$$
\begin{aligned}
& f_{k} \rightarrow f \text { strongly in } L^{2}\left(0, T ; V^{*}\right), \\
& w_{k} \rightarrow w \text { strongly in } W^{1,2}\left(0, T ; H^{1}(\Omega)^{n}\right), \\
& \dot{w}_{k} \rightarrow \dot{w} \text { strongly in } H \text { for every } t \in[0, T], \\
& \dot{z}_{k} \rightarrow L^{2}(0, T ; H) \text { strongly in } L^{2}(0, T ; H),
\end{aligned}
$$

using also (64) and (77), passing to the limit as $k \rightarrow \infty$ in (54), we obtain (39) by lower semicontinuity.

To prove (34), it is equivalent to show that there exists a set $N \subset[0, T]$ of measure zero such that for every $t_{i} \in[0, T] \backslash N$ with $t_{i} \rightarrow 0$, we have

$$
\dot{u}\left(t_{i}\right) \rightarrow u^{1} \text { strongly in } H
$$


(by Remark $2.12 \dot{u}(t)$ is a well-defined element in $H$ for every $t \in[0, T]$ ). By (28) and (39) we have

$$
\|\dot{u}(t)\|_{H}^{2}+\|\mathcal{E} u(t)\|_{H_{n}^{\mathbb{C}}}^{2} \leq\left\|u^{1}\right\|_{H}^{2}+\left\|\mathcal{E} u^{0}\right\|_{H_{n}^{\mathbb{C}}}^{2}+o(1), \text { as } t \rightarrow 0^{+}
$$

for every $t \in[0, T] \backslash N$ where $N \subset[0, T]$ is a set of measure zero. Now let $\left(t_{i}\right)$ be such that $t_{i} \in[0, T] \backslash N$ and $t_{i} \rightarrow 0$. By Remark 2.12, we already know that

$$
\dot{u}(t) \rightarrow \dot{u}(0) \quad \text { weakly in } H \text {, as } t \rightarrow 0^{+} \text {. }
$$

Moreover, by (68) together with (72) we can write for a.e. $t \in[0, T]$

$$
\dot{u}(t)-\dot{u}(0)=\int_{0}^{t} \ddot{u}(\tau) \mathrm{d} \tau=\lim _{k \rightarrow \infty} \int_{0}^{t} \dot{v}_{k}(\tau) \mathrm{d} \tau=\lim _{k \rightarrow \infty} v_{k}(t)-u^{1} \text { in } V_{0}^{*} ;
$$

hence, by choosing $t$ such that (up to subsequences) $v_{k}(t) \rightarrow \dot{u}(t)$ in $V_{0}^{*}$ (which is possible by (68)), we deduce that $\dot{u}(0)=u^{1}$ in $V_{0}^{*}$. Since both $\dot{u}(0)$ and $u^{1}$ are elements of $H$, and $H$ is embedded in $V_{0}^{*}$, we deduce $\dot{u}(0)=u^{1}$ in $H$. Therefore, the convergence (91) becomes

$$
\dot{u}\left(t_{i}\right) \rightarrow u^{1} \text { weakly in } H .
$$

This means that (89) is equivalent to

$$
\limsup _{i \rightarrow \infty}\left\|\dot{u}\left(t_{i}\right)\right\|_{H}^{2} \leq\left\|u^{1}\right\|_{H}^{2} .
$$

Since by (28) $t \mapsto \mathcal{E} u(t)$ is strongly continuous in $H_{n}$ we clearly have $\mathcal{E} u\left(t_{i}\right) \rightarrow \mathcal{E} u(0)$ strongly in $H_{n}$. By using the convergence (64) and a similar argument to (92) we deduce that $u(0)=u^{0}$, and hence that $\mathcal{E} u\left(t_{i}\right) \rightarrow \mathcal{E} u^{0}$ strongly in $H_{n}$. Therefore, by using $t_{i}$ in inequality (90) and passing to the limit as $i \rightarrow \infty$, we deduce exactly (93) and hence also (89).

Proposition 3.4 Let $u$ be the function given by Theorem 4.2, then $u$ satisfies condition (32) and the energy balance (38) for every $t$ Lebesgue point of $\|\dot{u}(\cdot)\|_{H}^{2}$.

Proof Let $w$ be the Dirichlet boundary condition considered in Definition 3.1. We note that for every $h \in(0, T)$ and for a.e. $t \in[0, T]$ the functions $u(t)-u(t-h)-(w(t)-w(t-h)) \in V_{t}$. Hence, if we define $z(t):=u(t)-w(t)$ we can test Eq. (33) with $\frac{z(t)-z(t-h)}{h}$ and integrate on $(h, T)$ to get

$$
\begin{aligned}
& \int_{h}^{t}\left\langle\ddot{u}(\tau), \frac{z(\tau)-z(\tau-h)}{h}\right\rangle_{\tau} \mathrm{d} \tau+\int_{h}^{t}\left\langle\mathbb{C} \mathcal{E} u(\tau), \frac{\mathcal{E} z(\tau)-\mathcal{E} z(\tau-h)}{h}\right\rangle_{H_{n}} \mathrm{~d} \tau \\
& \quad+\int_{h}^{t}\left\langle\mathbb{B} \mathcal{E} \dot{u}(\tau), \frac{\mathcal{E} z(\tau)-\mathcal{E} z(\tau-h)}{h}\right\rangle_{H_{h}} \mathrm{~d} \tau \\
& =\int_{h}^{t}\left\langle f(\tau), \frac{z(\tau)-z(\tau-h)}{h}\right\rangle_{\tau} \mathrm{d} \tau+\int_{h}^{t}\left\langle F(\tau), \frac{z(\tau)-z(\tau-h)}{h}\right\rangle_{\Theta} \mathrm{d} \tau .
\end{aligned}
$$

Since $u \in W^{1,2}(0, T ; V)$ and $w \in W^{2,2}(0, T ; H) \cap W^{1,2}(0, T ; V)$, we take the limit as $h \rightarrow 0^{+}$on both side of the previous equality

$$
\begin{aligned}
& \lim _{h \rightarrow 0^{+}} \int_{h}^{t}\left\langle\ddot{u}(\tau), \frac{z(\tau)-z(\tau-h)}{h}\right\rangle_{\tau} \mathrm{d} \tau+\int_{0}^{t}\langle\mathbb{C} \mathcal{E} u(\tau), \mathcal{E} \dot{z}(\tau)\rangle_{H_{n}} \mathrm{~d} \tau \\
& \quad+\int_{0}^{t}\langle\mathbb{B} \mathcal{E} \dot{u}(\tau), \mathcal{E} \dot{z}(\tau)\rangle_{H_{n}} \mathrm{~d} \tau=\int_{0}^{t}\langle f(\tau), \dot{z}(t)\rangle_{\tau} \mathrm{d} \tau+\int_{0}^{t}\langle F(\tau), \dot{z}(\tau)\rangle_{\Theta} \mathrm{d} \tau .
\end{aligned}
$$


In order to compute the limit of the first term in the left-hand side of (95), we use Lemma 2.16 to write

$$
\begin{aligned}
\int_{h}^{t}\left\langle\ddot{u}(\tau), \frac{z(\tau)-z(\tau-h)}{h}\right\rangle_{\tau} \mathrm{d} \tau= & \left\langle\dot{u}(t), \frac{z(t)-z(t-h)}{h}\right\rangle_{H}-\left\langle\dot{u}(h), \frac{z(h)-z(0)}{h}\right\rangle_{H} \\
& -\int_{h}^{t}\left\langle\dot{u}(\tau), \frac{\dot{z}(\tau)-\dot{z}(\tau-h)}{h}\right\rangle_{H} \mathrm{~d} \tau .
\end{aligned}
$$

Now, since $t$ is a Lebesgue point of $\|\dot{u}(\cdot)\|_{H}^{2}$ and $u$ satisfies the initial condition (34) we have

$$
\lim _{h \rightarrow 0^{+}}\left\langle\dot{u}(t), \frac{u(t)-u(t-h)}{h}\right\rangle_{H}=\|\dot{u}(t)\|_{H}^{2} \text { and } \lim _{h \rightarrow 0^{+}}\left\langle\dot{u}(h), \frac{u(h)-u(0)}{h}\right\rangle_{H}=\left\|u^{1}\right\|_{H}^{2} .
$$

Moreover, using the identity $\langle\dot{u}(\tau), \dot{u}(\tau)-\dot{u}(\tau-h)\rangle_{H}=-\frac{1}{2}\|\dot{u}(\tau-h)\|_{H}^{2}+\frac{1}{2}\|\dot{u}(\tau)\|_{H}^{2}+$ $\frac{1}{2}\|\dot{u}(\tau)-\dot{u}(\tau-h)\|_{H}^{2}$, we can write

$$
\begin{aligned}
\int_{h}^{t}\left\langle\dot{u}(\tau), \frac{\dot{u}(\tau)-\dot{u}(\tau-h)}{h}\right\rangle_{H} \mathrm{~d} \tau= & \frac{1}{2 h} \int_{t}^{t+h}\|\dot{u}(\tau)\|_{H}^{2} \mathrm{~d} \tau-\frac{1}{2 h} \int_{0}^{h}\|\dot{u}(\tau)\|_{H}^{2} \mathrm{~d} \tau \\
& +\frac{1}{2 h} \int_{h}^{t}\|\dot{u}(\tau)-\dot{u}(\tau-h)\|_{H}^{2} \mathrm{~d} \tau .
\end{aligned}
$$

Again using condition (34) and the fact that $t$ is a Lebesgue point of $\|\dot{u}(\cdot)\|_{H}^{2}$, we can write

$$
\begin{aligned}
\lim _{h \rightarrow 0^{+}} \int_{h}^{t}\left\langle\dot{u}(\tau), \frac{\dot{u}(\tau)-\dot{u}(\tau-h)}{h}\right\rangle_{H} \mathrm{~d} \tau= & \frac{1}{2}\|\dot{u}(t)\|_{H}^{2}-\frac{1}{2}\left\|u^{1}\right\|_{H}^{2} \\
& +\lim _{h \rightarrow 0^{+}} \frac{1}{2} \int_{h}^{t} \frac{\|\dot{u}(\tau)-\dot{u}(\tau-h)\|_{H}^{2}}{h} \mathrm{~d} \tau .
\end{aligned}
$$

By using the regularity assumption $w \in W^{2,2}(0, T ; H)$, a simple calculation leads to

$$
\begin{aligned}
\lim _{h \rightarrow 0^{+}} \int_{h}^{t}\left\langle\ddot{u}(\tau), \frac{w(\tau)-w(\tau-h)}{h}\right\rangle_{\tau} \mathrm{d} \tau & =\langle\dot{u}(t), \dot{w}(t)\rangle_{H}-\left\langle u^{1}, \dot{w}(0)\right\rangle_{H} \\
& -\int_{0}^{t}\langle\dot{u}(\tau), \ddot{w}(\tau)\rangle_{H} \mathrm{~d} \tau .
\end{aligned}
$$

Putting together (97) with (99), and using the fact that $z=u-w$, we can take the limit on both sides of(96) to get

$$
\begin{aligned}
& \lim _{h \rightarrow 0^{+}} \int_{h}^{t}\left\langle\ddot{u}(\tau), \frac{z(\tau)-z(\tau-h)}{h}\right\rangle_{\tau} \mathrm{d} \tau=\frac{1}{2}\|\dot{u}(t)\|_{H}^{2}-\frac{1}{2}\left\|u^{1}\right\|_{H}^{2} \\
& \quad+\lim _{h \rightarrow 0^{+}} \frac{1}{2} \int_{h}^{t} \frac{\|\dot{u}(\tau)-\dot{u}(\tau-h)\|_{H}^{2}}{h} \mathrm{~d} \tau+\frac{1}{2}\langle\dot{u}(t), \dot{w}(t)\rangle_{H} \\
& \quad-\frac{1}{2}\left\langle u^{1}, \dot{w}(0)\right\rangle_{H}+\frac{1}{2} \int_{0}^{t}\langle\dot{u}(\tau), \ddot{w}(\tau)\rangle_{H} \mathrm{~d} \tau .
\end{aligned}
$$


Putting together (95) with (100), we obtain for every Lebesgue point $t$ of $\|\dot{u}(\cdot)\|_{H}^{2}$

$$
\begin{gathered}
\frac{1}{2}\|\dot{u}(t)\|_{H}^{2}+\frac{1}{2}\|\mathcal{E} u(t)\|_{H_{n}^{\mathbb{C}}}^{2}+\int_{0}^{t}\|\mathcal{E} \dot{u}(\tau)\|_{H_{n}^{\mathbb{B}}}^{2} \mathrm{~d} \tau-\lim _{h \rightarrow 0^{+}} \frac{1}{2} \int_{h}^{t} \frac{\|\dot{u}(\tau)-\dot{u}(\tau-h)\|_{H}^{2}}{h} \mathrm{~d} \tau \\
=\frac{1}{2}\left\|u^{1}\right\|_{H}^{2}+\frac{1}{2}\left\|\mathcal{E} u^{0}\right\|_{H_{n}^{\mathbb{C}}}+\mathcal{W}_{\text {load }}(u ; 0, t)+\mathcal{W}_{\text {bdry }}(u ; 0, t) .
\end{gathered}
$$

But since we already know that $u$ satisfies the energy inequality (39), we immediately conclude that both condition (32) and the energy balance (38) hold.

Remark 3.5 At each time $t$ we expect that any energy increment for the solution $u(t)$, is due to the external forces. By identity (101), which is true for every $u$ satisfying (28)-(31) and (33), the requirement (32), i.e.,

$$
\lim _{h \rightarrow 0^{+}} \frac{1}{2} \int_{h}^{t} \frac{\|\dot{u}(\tau)-\dot{u}(\tau-h)\|_{H}^{2}}{h} \mathrm{~d} \tau=0,
$$

is natural in term of energy balance: indeed, the sum of the kinetic energy plus elastic energy plus viscous energy at time $t$ cannot exceed the amount of energy at time zero plus the work done by the external forces in the time interval $[0, t]$.

Remark 3.6 Since $\dot{u}(t)-\dot{w}(t) \in V_{t}$ for a.e. $t \in[0, T]$, we can use it as test function in (33) and integrate on $(0, t)$ to obtain

$$
\begin{gathered}
\int_{0}^{t}\langle\ddot{u}(\tau), \dot{u}(\tau)\rangle_{\tau} \mathrm{d} \tau+\frac{1}{2}\|\mathcal{E} u(t)\|_{H_{n}^{\mathbb{C}}}^{2}+\int_{0}^{t}\|\mathcal{E} \dot{u}(\tau)\|_{H_{n}^{\mathbb{B}}}^{2} \mathrm{~d} \tau \\
=\frac{1}{2}\left\|\mathcal{E} u^{0}\right\|_{H_{n}^{\mathbb{C}}}^{2}+\mathcal{W}_{\text {load }}(u ; 0, t)+\mathcal{W}_{\text {bdry }}(u ; 0, t) .
\end{gathered}
$$

Comparing this last identity with the energy balance (38), we have for a.e. $t \in(0, T)$ that

$$
\frac{1}{2}\|\dot{u}(t)\|_{H}^{2}-\frac{1}{2}\left\|u^{1}\right\|_{H}^{2}=\int_{0}^{t}\langle\ddot{u}(\tau), \dot{u}(\tau)\rangle_{\tau} \mathrm{d} \tau
$$

and since $\tau \mapsto\langle\ddot{u}(\tau), \dot{u}(\tau)\rangle_{\tau} \in L^{1}(0, T)$ we deduce that $\|\dot{u}(\cdot)\|_{H}^{2} \in W^{1,1}(0, T)$.

Putting together Theorem 3.2 and Proposition 3.4, we deduce the existence of a solution $u$ to the damped system of elastodynamics. Moreover, using (101) we can also obtain the uniqueness of weak solutions considered in Definition 3.1. This is the content of the next theorem.

Theorem 3.7 (Existence and uniqueness) Under hypothesis of Theorem 3.2, there exists a unique solution u considered in Definition 3.1. Moreover, $u$ satisfies the energy balance

$$
\begin{aligned}
& \frac{1}{2}\|\dot{u}(t)\|_{H}^{2}+\frac{1}{2}\|\mathcal{E} u(t)\|_{H_{n}^{\mathbb{C}}}^{2}+\frac{1}{2} \int_{0}^{t} \frac{1}{2}\|\mathcal{E} \dot{u}(\tau)\|_{H_{n}^{\mathbb{B}}}^{2} \mathrm{~d} \tau \\
& =\frac{1}{2}\left\|u^{1}\right\|_{H}^{2}+\frac{1}{2}\left\|\mathcal{E} u^{0}\right\|_{H_{n}^{\mathbb{C}}}+\mathcal{W}_{\text {load }}(u ; 0, t)+\mathcal{W}_{\text {bdry }}(u ; 0, t)
\end{aligned}
$$

for a.e. $t \in[0, T]$. 
Proof The existence of a solution satisfying (102) is simply a consequence of Theorem 3.2 and Proposition 3.4.

To show uniqueness, we notice that (28)-(33) are all preserved under linear combinations. Therefore, the difference $v$ between two solutions is a solution with Dirichlet and Neumann homogeneous conditions, with forcing term $f=0$, and satisfying $v(0)=0$ and

$$
\lim _{h \rightarrow 0^{+}} \frac{1}{h} \int_{0}^{h}\|\dot{v}(t)\|_{h}^{2} \mathrm{~d} t=0 .
$$

Moreover, using the same argument as in Proposition 3.4, since (32) holds for $v$, we have

$$
\frac{1}{2}\|\dot{u}(t)\|_{H}^{2}+\frac{1}{2}\|\mathcal{E} u(t)\|_{H_{n}^{\mathbb{C}}}^{2}+\frac{1}{2} \int_{0}^{t} \frac{1}{2}\|\mathcal{E} \dot{u}(\tau)\|_{H_{n}^{\mathbb{B}}}^{2} \mathrm{~d} \tau=0
$$

for a.e. $t \in[0, T]$. Therefore, $\dot{v}(t)=0$ a.e. on $[0, T]$. Since $v \in W^{1, \infty}(0, T ; H)$ and $v(0)=0$, we conclude $v(t)=0$ a.e. on $[0, T]$.

Finally, one can also prove that the energy balance (38) holds for every $t \in[0, T]$ and that the map $t \mapsto \dot{u}(t)$ is strongly continuous in $H$. For the proof of this result we refer to [7, Lemma 3.10].

Proposition 3.8 Under the assumptions of Theorem 3.2, let $u$ be the weak solution of the damped wave equation considered in Definition (3.1), with initial conditions $u(0)=u^{0}$ and (34). Then $t \mapsto \dot{u}(t)$ is continuous from $[0, T]$ to $H$ and the energy balance (38) holds for every $t \in[0, T]$.

\section{The undamped system of elastodynamics}

In this section, we study weak solutions of the undamped system of elastodynamics

$$
\ddot{u}(t)-\operatorname{div}[\mathbb{C} \mathcal{E} u(t)]=f(t) .
$$

As for the damped case, we give the definition of weak solution.

Definition 4.1 Assume (a), (b), (c) and (d). With the notation introduced in Sect. 2, let $f \in$ $W^{1,2}\left(0, T ; V^{*}\right)$, let $w \in W^{2,2}(0, T ; H) \cap W^{1,2}\left(0, T ; H^{1}(\Omega)^{n}\right)$ and let $F \in W^{1,2}\left(0, T ; N_{\Theta}\right)$ where $\Theta$ is the function relative to the crack set $\Gamma$ given by Theorem 2.5 . We say that $u$ is a weak solution of (1) on the time dependent domain $t \mapsto \Omega \backslash \Gamma(t)$ with Dirichlet boundary condition $w(t)$ on $\partial_{D} \Omega$, Neumann boundary condition $F(t)$ on $\partial_{N} \Omega$, and homogeneous Neumann boundary condition on $\Gamma(t)$, if

$$
\begin{aligned}
& u \in L^{\infty}(0, T ; V) \cap W^{1, \infty}(0, T ; H) . \\
& \text { For every } t \in[0, T] u(t)-w(t) \in V_{t} . \\
& \text { For every } s \in[0, T) u \in W^{2,2}\left(s, T ; V_{s}^{*}\right) \text { and } \\
& \left\|P_{s t} \ddot{u}(t)\right\|_{s}^{*} \leq g(t) \text { for a.e. } t \in(s, T), \text { for some } g \in L^{2}(0, T) .
\end{aligned}
$$

For a.e. $t \in[0, T]$

$\langle\ddot{u}(t), \phi\rangle_{t}+\langle\mathbb{C} \mathcal{E} u(t), \mathcal{E} \phi\rangle_{H_{n}}-\langle F(t), \operatorname{Tr}(\phi)\rangle_{\Theta}=\langle f(t), \phi\rangle_{t}$, for every $\phi \in V_{t}$

where $\ddot{u}(t)$ is the one given by Definition 2.15. 
Given $u^{0} \in V$ such that $u^{0}-w(0) \in V_{0}$ and $u^{1} \in H$, since $t \mapsto u(t)$ is strongly continuous in $H$ the initial value for $u$ is well defined as element of $H$. Moreover, we are able to prescribe the initial conditions, respectively, for $\mathcal{E} u(0)$ and $\dot{u}(0)$ by asking

$$
\lim _{h \rightarrow 0^{+}} \frac{1}{h} \int_{0}^{h}\left\|\mathcal{E} u(t)-\mathcal{E} u^{0}\right\|_{H_{n}}^{2} \mathrm{~d} t=0,
$$

and

$$
\lim _{h \rightarrow 0^{+}} \frac{1}{h} \int_{0}^{h}\left\|\dot{u}(t)-u^{1}\right\|_{H}^{2} \mathrm{~d} t=0 .
$$

Since in this case $\dot{u}(t)$ is in general only an element of $H$, we need to consider also a weakened formulation of the work due to the Neumann boundary conditions. More precisely, the term appearing in the work due to the boundary forces $\mathcal{W}_{\text {bdry }}\left(u ; t_{1}, t_{2}\right)$, which in the damped case read as

$$
\int_{t_{1}}^{t_{2}}\langle F(t), \dot{u}(t)\rangle_{\Theta} \mathrm{d} t
$$

becomes

$$
\left\langle F\left(t_{2}\right), u\left(t_{2}\right)\right\rangle_{\Theta}-\left\langle F\left(t_{1}\right), u\left(t_{1}\right)\right\rangle_{\Theta}-\int_{t_{1}}^{t_{2}}\langle\dot{F}(t), u(t)\rangle_{\Theta} \mathrm{d} t,
$$

for every time interval $\left[t_{1}, t_{2}\right] \subset[0, T]$.

The following is the main result.

Theorem 4.2 Assume (a), (b), (c) and (d). Let $f, w$ and $F$ be as in Definition 4.1. Then, given two initial conditions $u^{0} \in V$ such that $u^{0}-w(0) \in V_{0}$ and $u^{1} \in H$, there exists a solution $u$ of (1) with initial conditions (109) and (110). Moreover, $u$ satisfies the energy inequality

$$
\frac{1}{2}\|\dot{u}(t)\|_{H}^{2}+\frac{1}{2}\|\mathcal{E} u(t)\|_{H_{n}^{\mathbb{C}}}^{2} \leq \frac{1}{2}\left\|u^{1}\right\|_{H}^{2}+\frac{1}{2}\left\|\mathcal{E} u^{0}\right\|_{H_{n}^{\mathbb{C}}}+\mathcal{W}_{\text {load }}(u ; 0, t)+\mathcal{W}_{\text {bdry }}(u ; 0, t)
$$

for a.e. $t \in[0, T]$.

Proof Since the argument is similar to the one given for Theorem 4.2, we simply give a sketch of the proof.

For $k \in \mathbb{N}$, we set $\tau_{k}:=T / k$ and $t_{k}^{j}:=j \tau_{k}$. For $j=1,2, \ldots, k$ we define $f_{k}^{j} \in V^{*}$ and $w_{k}^{j} \in H^{1}(\Omega)^{n}$ by

$$
f_{k}^{j}:=f\left(t_{k}^{j}\right), \quad w_{k}^{j}:=w\left(t_{k}^{j}\right)
$$

using that $f \in W^{1,2}\left(0, T ; V^{*}\right)$ and $w \in W^{1,2}\left(0, T ; H^{1}(\Omega)^{n}\right)$, so $f$ and $w$ are well-defined elements of $V^{*}$ and $H^{1}(\Omega)^{n}$, respectively, for every $t \in[0, T]$. Inductively, we define $u_{k}^{j}$ for $j=-1,0, \ldots, k$ by the following

$$
u_{k}^{0}:=u^{0}, \quad u_{k}^{-1}:=u^{0}-\tau_{k} u^{1} ;
$$

then, for $j=0,1, \ldots, k-1$, the function $u_{k}^{j+1}$ is the minimizer in $V_{t_{k}^{j+1}}+w_{k}^{j}$ of

$$
u \mapsto\left\|\frac{u-u_{k}^{j}}{\tau_{k}}-\frac{u_{k}^{j}-u_{k}^{j-1}}{\tau_{k}}\right\|_{H}^{2}+\|\mathcal{E} u\|_{H_{n}^{\mathbb{C}}}^{2}-2\left\langle f_{k}^{j}, u\right\rangle_{t_{k}^{j+1}}
$$


Then if we define $u_{k}, \tilde{u}_{k}, w_{k}, \tilde{w}_{k}, v_{k}$, and $z_{k}$ as in (49)-(53), then proceeding exactly as in 3.2, we deduce the following bounds

$$
\begin{aligned}
& \mathcal{E} u_{k}(t) \text { and } \mathcal{E} \tilde{u}_{k}(t) \text { are bounded in } H_{n} \text { uniformly in } t \text { and } k, \\
& \dot{u}_{k}(t) \text { and } v_{k}(t) \text { are bounded in } H \text { uniformly in } t \text { and } k, \\
& u_{k}(t) \text { is bounded in } V \text { uniformly in } t \text { and } k \text {. }
\end{aligned}
$$

Furthermore, using the Euler equation for $u_{k}^{j+1}$, we can write for all $t \in\left(t_{k}^{j}, t_{k}^{j+1}\right)$

$$
\left\langle\dot{v}_{k}(t), \phi\right\rangle_{H}+\left\langle\mathbb{C} \mathcal{E} \tilde{u}_{k}(t), \mathcal{E} \phi\right\rangle_{H_{n}}=\left\langle f_{k}(t), \phi\right\rangle_{t_{k}^{j+1}}
$$

for every $\phi \in V_{t_{k}^{j+1}}$. The last equation leads us to write for all $t \in\left(t_{k}^{j}, t_{k}^{j+1}\right)$

$$
\left\|\dot{v}_{k}(t)\right\|_{t_{k}^{j+1}}^{*} \leq\left\|\mathcal{E} \tilde{u}_{k}(t)\right\|_{H_{n}^{\mathbb{C}}}+\left\|f_{k}(t)\right\|_{t_{k}^{j+1}}^{*} .
$$

In particular, fix $s \in[0, T)$, then for every $t_{1}, t_{2} \in[s, T]$ with $t_{1}<t_{2}$, we have

$$
\int_{t_{1}}^{t_{2}}\left\|P_{s t} \dot{v}_{k}(t)\right\|_{s}^{*} \mathrm{~d} t \leq \int_{t_{1}}^{t_{2}}\left(\left\|\mathcal{E} \tilde{u}_{k}(t)\right\|_{H_{n}^{\mathbb{C}}}+\left\|f_{k}(t)\right\|_{s}^{*}\right) \mathrm{d} t .
$$

Again following exactly 3.2, we deduce that up to subsequences

$$
\begin{aligned}
& u_{k} \rightarrow u, \text { weakly in } W^{1,2}(0, T ; H), \\
& \tilde{u}_{k} \rightarrow u, \text { weakly in } L^{2}(0, T ; V), \\
& v_{k} \rightarrow v \text { weakly in } L^{2}(0, T ; H), \\
& v_{k} \rightarrow v \text { weakly in } W^{1,2}\left(s, T ; V_{s}^{*}\right) .
\end{aligned}
$$

Moreover, $u \in L^{\infty}(0, T ; V) \cap W^{1, \infty}(0, T ; H), \dot{u}(t)=v(t)$ a.e. on $[0, T]$, and

$$
\left\|P_{s t} \ddot{u}(t)\right\|_{t}^{*} \leq M+\|f(t)\|_{s}^{*},
$$

for every $s \in[0, T)$ and for a.e. $t>s$.

Now the proof that $u$ is a solution, and that satisfies the energy inequality (112) proceeds as in the damped case. Finally, it remains to prove that $u$ satisfies the initial conditions (109) (110). It is enough to show that there exists a set $N \subset[0, T]$ of measure zero such that for every $t_{i} \in[0, T] \backslash N$ with $t_{i} \rightarrow 0$, we have

$$
\dot{u}\left(t_{i}\right) \rightarrow u^{1} \text { strongly in } H,
$$

and

$$
\mathcal{E} u\left(t_{i}\right) \rightarrow \mathcal{E} u^{0} \text { strongly in } H_{n} .
$$

Again this can be achieved following a similar argument to the damped case.

Acknowledgements The author wishes to thank Prof. Gianni Dal Maso for many helpful discussions on the topic.

\section{References}

1. Ambrosio, L., Fusco, N., Pallara, D.: Functions of Bounded Variation and Free Discontinuity Problems. Oxford University Press, Oxford (2000) 
2. Caponi, M., Lucardesi, I., Tasso, E.: Energy dissipation balance of a smooth moving crack. J. Math. Anal. Appl. (2019). https://doi.org/10.1016/j.jmaa.2019.123656

3. Chambolle, A., Conti, S., Iurlano, F.: Approximation of functions with small jump sets and existence of strong minimizers of Griffith's energy. J. Math. Pures Appl. 128, 119-139 (2019)

4. Conti, S., Focardi, M., Iurlano, F.: Existence of strong minimizers for the Griffith static fracture model in dimension two. Annales de l'Institut Henri Poincaré C, Analyse non linéaire 36(2), 455-474 (2018)

5. Dal Maso, G.: Generalised functions of bounded deformation. J. Eur. Math. Soc. 15(5), 1943-1997 (2013)

6. Dal Maso, G., Francfort, G.A., Toader, R.: Quasistatic crack growth in finite elasticity. Preprint SISSA, Trieste, (2004). http://www.sissa.it/fa/

7. Dal Maso, G., Larsen, C.J.: Existence for wave equations in domains with arbitrary growing cracks. Atti Accad. Naz. Lincei Rend. Lincei Mat. Appl. 22(3), 387-408 (2011)

8. Dal Maso, G., Larsen, C.J., Toader, R.: Existence for constrained dynamic Griffith fracture with a weak maximal dissipation condition. J. Mech. Phys. Solids 95, 697-707 (2016)

9. Dal Maso, G., Lazzaroni, G.: Quasistatic crack growth in finite elasticity with non-interpenetration. Annales de l'Institut Henri Poincaré (C) Non Linear Analysis 27(1), 257-290 (2010)

10. Dal Maso, G., Toader, R.: A model for the quasi-static growth of brittle fractures: existence and approximation results. Arch. Ration. Mech. Anal. 162(2), 101-135 (2002)

11. Federer, H.: Geometric Measure Theory. Springer, Berlin (1969)

12. Francfort, G.A., Larsen, C.J.: Existence and convergence for quasi-static evolution in brittle fracture. Commun. Pure Appl. Math. 56(10), 1465-1500 (2003)

13. Freund, L.B.: Dynamic Fracture Mechanics. Cambridge University Press, New York (1990)

14. Larsen, C.J.: Models for dynamic fracture based on Griffith's criterion. In: Hack1, K. (ed.) IUTAM Symposium on Variational Concepts with Applications to the Mechanics of Materials, vol. 21, pp 131140. Sringer, Berlin (2010)

15. Tasso, E.: On the continuity of the trace operator in $G S B V(\Omega)$ and $G S B D(\Omega)$. Accepted on ESAIM: COCV (2019). https://doi.org/10.1051/cocv/2019014

Publisher's Note Springer Nature remains neutral with regard to jurisdictional claims in published maps and institutional affiliations. 